I NTER NATIONAL MONETARY FUND

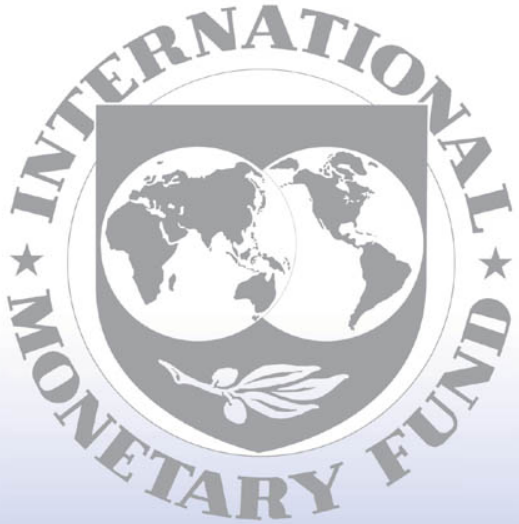

Staff

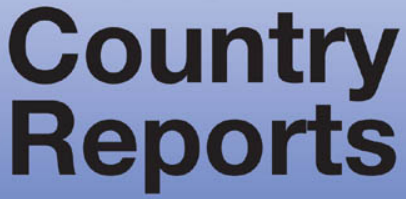


- 1995 International Monetary Fund

February 1995

IMF Staff Country Report No. 95/17

\section{Tunisia-Statistical Annex}

This statistical annex on Tunisia was prepared by a staff team of the International Monetary Fund as background documentation for the periodic consultation with this member country. In releasing this document for public use, confidential material may have been removed at the request of the member.

Copies of this report are available to the public from

International Monetary Fund - Publication Services

700 19th Street, N.W. - Washingtoo, D.C. 20431

Telephone: (202) 623-7430 - Telefax: (202) 623-7201

Telex (RCA): 24833I IMF UR

Internet: pubserv@imf.org

Price: $\$ 15.00$ a copy

International Monetary Fund

Washington, D.C.

(CInternational Monetary Fund. Not for Redistribution 
This page intentionally left blank

(C) International Monetary Fund. Not for Redistribution 


\section{INTERNATIONAL MONETARY FUND}

TUNISIA

\section{Statistical Annex}

Prepared by a staff tean consisting of $\mathrm{Mr}$. A. Jbili (head-MED), Mr. K. Enders (MED), Mr. S. Chami (MED), Mr. P. Duran (MED), and Ms. A.M. Gulde (MAE)

Approved by the Middle Eastern Department

December 22, 1994

Contents

Page

Basic Data iii

Recent Progress in Financial Sector Reform 1

\section{$\underline{\underline{\text { Tables }}}$}

1. Real GDP Growth by Sector, 1990-94 16

2. Supply and Use of Resources--Real Growth Rates, 1990-1994 17

3. Sectoral Distribution of GDP, 1989-94

4. Supply and Use of Resources, 1989-94 18

5. Production of Major Agricultural Crops, 1989-94 20

6. Supply and Use of Cereals, 1989/90-1993/94 21

7. Producer Prices of Principal Agricultural Commodities, $1989-94$

8. Energy Production and Consumption, 1989-94 23

9 . Indicators of Tourism Activity, 1989-94 24

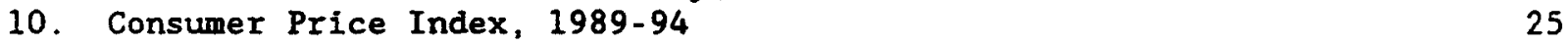

11. Consumer Price Index by Price Regime, 1989-93 26

\begin{tabular}{ll} 
12. Producer Price Index, 1989-94 & 27 \\
\hline
\end{tabular}

\begin{tabular}{ll}
13. & Wage and Employment Indicators, 1989-93 \\
\hline
\end{tabular}

14. Gross Fixed Investment by Economic Sector and Financing, 1989.94

15. Consolidated Financial Operations of the Central Government, 1989-94

16. Consolidated Revenue and Grants of the Central

Government, 1989-93

17. Government Petroleum Revenues, $1989-94$

18. Economic Classification of Consolidated Expenditure of the Central Government, 1989-94 
20. Monetary Survey, $1989-94$

21. Assets and Llabllitles of the Central Bank, 1989-94 36

22. Assets and Llabilitles of Deposit Money Banks, 1989-94 37

23. Selected Interest Rates, 1989-94 38

24. Balance of Payeents, 1989-94 39

25 . Foreign Trade Indicators, 1990-94 40

26. Velue of Forelon Trede by Coreodity Clase, 1989-94 41

27. Volume of Forelgn Trede by Comodity Cless, 1990-94 42

28 . Trade Balance in Hydrocarbons, 1989-93 43

29. Exports of Phosphate Rock, Phosphate Derivatives, and Other Chenical Products, 1989-93

30. . Exports and Imports of Primary Products by Major Categories, 1989-93

31. Direction of Trede, 1989.93

32. External Debt and Debt Service Payeants, 1989.93 47

33. Cosposition of External Debt by Creditor, 1989-93 48

34. Selected Exchange Rate Indlces, 1989-94 49

35. Structure of Imports by Reg1me, $1989.92 \quad 50$

\section{Charts}

1. Financial Deepening

2. Troasury Bille Outstanding

3. Nunber of Investrent Funds

\begin{tabular}{lr} 
4. Stock Market Capitalization & 10 \\
\hline
\end{tabular}

S. Stock Market Developeent 
Tuniaia - Bric Data

Area, population and GDP per capita

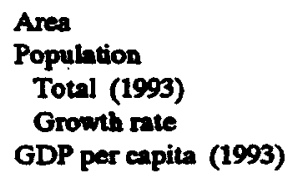

Gross domeatic product at 1950 market prices

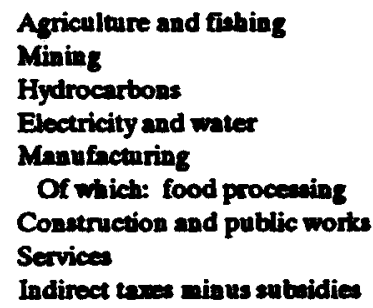

Gross national product

Gross mational dieponeble income

Gross national savings

Prices (percentage changes)

GDP deflator

Consumer prices

Government fiaance ?

\author{
165,154 square kilometers \\ 8.5 million \\ 1.8 percent \\ SDR 1,235
}

Pnel.

1989 1990

1991

1992

1993

1994

(in millions of dinars)

$\begin{array}{rrrrrr}10,019 & 10,798 & 11,217 & 12,110 & 12,365 & 12,906 \\ 1,305 & 1,763 & 2,019 & 2,128 & 2,003 & 1,864 \\ 125 & 103 & 103 & 99 & 86 & 98 \\ 677 & 620 & 706 & 707 & 634 & 619 \\ 213 & 215 & 225 & 237 & 247 & 256 \\ 1,763 & 1,796 & 1,883 & 2,026 & 2,079 & 2,241 \\ 438 & 394 & 430 & 480 & 442 & 486 \\ 376 & 429 & 426 & 453 & 492 & 531 \\ 4,431 & 4,643 & 4,601 & 5,099 & 5,405 & 5,792 \\ 1,130 & 1,2229 & 1,254 & 1,361 & 1,418 & 1,505\end{array}$

$\begin{array}{rrrrrr}9,531 & 10,798 & 11,997 & 13,754 & 14,688 & 16,134 \\ 6,520 & 7,390 & 7,990 & 8,932 & 9,586 & 10,538 \\ 1,157 & 1,245 & 1,444 & 1,590 & 1,769 & 1,943 \\ 2,157 & 2,635 & 2,912 & 3,635 & 4,168 & 4,658 \\ 101 & 242 & 231 & 496 & 140 & 82 \\ -404 & -714 & -580 & -899 & -974 & -1,087 \\ 9,142 & 10,438 & 11,498 & 13,236 & 13,926 & 15,445 \\ 9,591 & 10,943 & 12,013 & 13,736 & 14,500 & 16,101 \\ 1,914 & 2,308 & 2,579 & 3,214 & 3,145 & 3,620\end{array}$

$\begin{array}{ll}7.2 & 5.1\end{array}$

6.2

4.6

5.2

7.7

6.5

7.0
8.2

5.8

4.0

4.5

$\begin{array}{rr}2,928 & 3,286 \\ 2,258 & 2,551 \\ 670 & 735 \\ 143 & 70 \\ 3,483 & 3,787 \\ 2,693 & 2,863 \\ 725 & 880 \\ 65 & 44 \\ -412 & -431 \\ 118 & 75 \\ 294 & 356 \\ 45 & 85\end{array}$

$$
\begin{array}{r}
3,491 \\
2,875 \\
617 \\
33 \\
4,179 \\
3,176 \\
1,026 \\
-24 \\
-655 \\
319 \\
336 \\
45
\end{array}
$$

4,451

4,486

4,044 
Teriain - Basic Data (concluded)

1989

1990

1991

1992

1993

Prel.

(le millions of dinare)

Money and credit (end of period)

Foreign senets (net)

Domestic credit

Central government (net)

Other ageats

Money and quan - money

Money

Quasi-money

Oher items (set)
668

5,563

722

4,841

4,910

2,527

2,383

1,321

612
6,143
808
5,335
5,220
2,649
2,571
1,535

526
6,741

853

5,888

5,507

2,640

2,867

1,761
June

$\begin{array}{rr}547 & 563 \\ 7,478 & 8,004 \\ 687 & 640 \\ 6,791 & 7,364 \\ 5,903 & 6,294 \\ 2,781 & 2,916 \\ 3,122 & 3,378 \\ 2,122 & 2,273\end{array}$

400

8,298

507

7,791

6,216

2,954

3,262

2,482

(In millions of SDRs)

Balance of paymeats

\section{Exports, fo.b. \\ Imports, fo.b. \\ Trade balance \\ Services and transfen (net) \\ Current accoust bulasce \\ Capitul sccount (net) \\ Grants \\ Direct foreign inventment (aet) \\ Medium- and long-verm loans \\ Other \\ Overall surplus or deficit (-)}

Grose official reserves

(end of period)

Excernal debt

Disbursed and ouratanding (end of period)

Terms of trade (percentage changes)

Export prices (in SDRs)

Import prices (in SDRs)

Terms of trade

Exchange rates (period averages)

Dinar/SDR

Dinar/U.S. dollar

Nominal effective exchange rate

(perceutage changes)

Real effective exchange rate

(percentage changes)

$\begin{array}{rr}2,286 & 2,592 \\ -3,223 & -3,828 \\ -942 & -1,236 \\ 699 & 735 \\ -242 & -500 \\ 305 & 426 \\ 161 & 151 \\ 113 & 137 \\ 101 & 80 \\ -70 & 55 \\ 62 & -7\end{array}$

739

565

559

658

627

778

4,713

5,007

5,355

5,424

5,637

6,157

7.9

6.7

1.1

1.217

0.949

1.192

0.878

1.265

0.925

1.246

0.884

1.402

1.004

0.7

$-2.0$

$-1.7 \quad-2.8$
$-23$

1.1

\section{9}

$-2.1$

-4.7
-5.0

0.4

$-0.5$

0.3

$-0.8$

$\begin{array}{rrrrr}-4.2 & -4.6 & -2.3 & 0.7 & -2.0 \\ -1.7 & -2.8 & 1.1 & 2.1 & -1.5\end{array}$

1.451 1.016 


\section{Becent Progress in Flnanchel Sector Refor}

Since 1987. Tunisia has undertaken a series of masures of financial sector liberalization and reform. The current appendix sumarizes the progress achieved, highlighting in particular the anin changes introduced over the past two years. These include the adoption of a new banking law, the introduction of an interbank forelgn exchange anket, the lssunce of negotiable treasury bills, and the revision of the stock market law.

\section{Backeround $\mathcal{L}$}

At the start of the reform process in 1987, the Tunlsian banking systen was characterized by a prollferation of restriction, including full controls on lending and doposit interest rates, directed credit, Ilquid asset ratios, and Central Bank (BCT) control over lendias dacieions. This, together with the lack of competitive practices, left nost banking institutions with weak asset bases. Forelgn competition in comerclal banking was also abeent. The raninder of the financiel sector was relatively sall and, for the nost part, also tightly controlled. This applied In particular to the developent banks and Insurance companies, which--like comerclal banks--were obliged to hold long-tern public dabt ("bons d'equipenent") at below enket interest rates. While, de jure, a stock ankket existed since 1969, it was virtually inactive.

Since the ald 1980s, the Increasing costs of an Inefficlent banking sector and the attendant problen for monetary control created a nounting inpetus for reform. The changes initiated in 1987 and 1988 alad at liberalizing interest rates and allowing ancket forces to play a greater role in banks' business, while at the sase tise creating a deeper and nore diversifled financial maxket. In parallel, significant inprovenonts in the prudential eupervision were inplenented. By 1993, visible progress had been ande. The banking sector had been liberated fron the nost restrictive features, although soen riglditles reanined and a lack of competition still hindered efficiency and profitability. 2/ The nonbank financial sector, In particular the Imvestont funds (SICAPs and SICAVs) and the stock anket, showed strong growth, albelt from a low base. The stock arket, hovever,

1 For a survey of previous neasures and results see Nsouli, Saleh M., Sena Eken, Paul Duran, Gerwin Bell, and 2uhta Yucelik: "The Path to Convertiblifty and Growth. - The Tunisian Experience," IMF decentenl Paper Ne.109, Decenber 1993. A chronology of all iuportant neasures taken since 1987 is provided in the attachent.

2) Restrictions in place included controls on average lending rates, prescribed naximu deposit rates for short-term deposits and rates for special savings accounts, and lending requirenents for priority sectors (agriculture, exports, and saall and eediue enterprises) and preferential lending rates for the same sectors. In addition, the Central Bank continued to guide its refinancing according to sectoral goals. 
suffered fron a weak supply of equity shares and the lack of an approprlate settlement syster.

\section{The refon nensures in 1993 and 1994}

Against this background, the authorities ande further beacway in financlal sector reform in 1993 and 1994.

\section{a. The ney brokting lay}

In an effort to strengthen banks' role in the econory, the new banking law, which was passed in Fobruary 1994, Introduces "universel banking" and pernite deposit money banks to expand their activities to now areas, wch as regular nediw- and long-tern lending, portfollo managenent, and flancial restructuring services. Developent banks are also allowed to axtend shortterm credit to enterprises for which they have provided investiont financing. The opening-up of such activities to banke ins at enbancing their profitability by allowing for econosies of scale and diveraffication, whlle helping introduce now financlal products. Finally, the now law creates a new category of financlal instltutions, the Imvestont bank (banque d'effalres), specialized in providing a wide range of imvestuntreleted services.

To ensure stab121ey of the financial systen, the law requires a flrmer prudential overalght of comercial banks by the BCT. To that effoct, it reaffiras and codifles a range of prudantial requiranante, including ainime capital standarde (dinar 10 alliton for comercial banke and dinar 3 allion for the newly introduced "banque d'affaires"); a requirement of atching the naturities between assets and liablilties; a capital-asset rat10; prudential 11quidity ratios; IIntes on loan and invastant concentration; overaight over bank nergers and sizeable participation in banks; and prudential control over the opening and closing of branches. In addition, the law explicitly states that banks are no longer required to acquire public debt inetruments. Also, the supervisory powers of the BCT have been strengthened to Include on-site Inapections and extermal audits. In the case of noncompliance with the law, the banks any be subject to a wide range of sanctions, Including a withdrawal of the banking license, as well as disciplinary actions against managenent or mabers of the banks" board.

\section{b. The interbank foreten exchange narket}

An interbank forelgn exchinge market was established in March 1994 following technical assistance providad by MAE. Its introduction marked a further step toward financial liberalizetion and strengthening of the banking sector. I In the naw systen, and during a transition period, the

1 Prior to March 1994, the banks were not allowed to trade in foreign exchange among thenselves. 
BCI continues to announce a central rate, around which banks trade foreign exchange freely anong thenselves within band of 1 percent on either $s i d$. Only comercial and development banks have been so far allowed to operate in the interbank market.

As a counterbalance to banks' Increased freedon in forelgn exchange transactions, the BCT has instituted etrict regulations dasigad to prevent excess exposure of banks to forelgn currency riske and to protect anall custoners. Concerning prudential regulations, open positions nut not exceed 5 percent of the bank's capital in each currancy and 20 percent in all currencies combined; any excess above these linits has to be sold at the end of the day to the BCT ("nivellenent") at Its central rate. To protect customers, banks are required to post buying and selling rates with a maximun spread of 0.25 percent.

During the few wonths of its operation, the interbank arket has functioned swoothly with a fairly large particlpation of banks. About 70 80 percent of all foreign exchange tranaction now take place in the interbank market, representing an average dally volue of $60-70$ alllion dinars. Transactions conducted at the central bank rate nosty irvolve goverment operations and those denoninated in currencles for which no sufficient interbank activity exists. Most foreign exchange deals have been directly related to current transactions, while those involving nore "sophisticated" financial instruments--such as swaps, reverse repurchase agreements or other forns of derivatives-have been all but absent. Although competition anong banks has ranaland relatively linited, it has recently intensified as experience in the narket is gainod and the necessary technical and human capital is acquired. $W$

\section{c. Treasury bills and bonds}

Treasury bills (TBs) were first introduced in 1989 and nodified on several occasions to becone an important sevings instrunent. Three institutional features contributed to the rapid success: (1) Treasury bills were auctioned to banks and subscriptions vere counted toward fulfilling the requirement of holding treasury paper ("ratio d'aplol en effets publics"); (ii) banks were pernitted to sell TBs to the public at a slightly lower interest rate, thus providing then with a financial incentive to place a large proportion of TBs with the public; and (111) public deand was high, because banks guaranteed the liquidity without discount of the instrumens at any moment ("cessibllite"), thus aaking TBs an attractive altermative to tine and savings deposits. $2 /$ Yet this arrangement, in particular the "cessibilité," has hindered the energence of a secondary narket, since TBs were practically fully liquid and could be redeaned at face value. $3 /$

1) Most banks have established "trading rooms" with modern electronic equipments and trained their personnel in forelgn exchange operations.

2) The Treasury bills are sometines referred to as "bons cessibles."

3) The banks, however, charge a suall redeuption fee. 
To address some of these issues, the Tunisian authorities introduced in late 1993 negotiable treasury bonds (NTBs) ("bons du Trésor negotiables"), which can be traded at the stock market. Those instruments are to fulfill three functions not achieved by the TBs: (i) supply a true long-term instrument, (ii) assist the development of a secondary market, and (iii) allow for a market-determined yield curve. The NTBs and the TBs are to coexist, giving custoners a choice between the highly liquid instrunents (TBs) and longer-term instruments traded at the stock exchange (NTBs). 1

With only limited working experience, the share of NTBs in total governnent domestic debt has remained limited. $2 /$ After only two issuances at the stock market, investors' interest seems to have faded, reflecting technical difficulties and inadequate design, including the segmented character of each issue. It appears also that the interest rate, which is almost identical to that of TBs, does not reflect the longer maturity and the lesser liquidity of NTBs. In addition, the secondary market suffers from weaknesses in the settlement system, in fact 1 imiting the transferability and liquidity of the new bonds. At present, the authorities are examining the institutional and technical set-up, with a view to improving both instrument design and the settlement system in the near future. $3 /$

\section{d. The stock market}

The adoption of the stock market law on November 14, 1994 was another important building block in the financial sector reform. A stock exchange had been in existence since the late 1960s, but its growth was constrained by a number of factors, including the limited supply of equity, the dominant role of the banks in raising capital, and the absence of an efficient settlement system. In addition, the bourse was publicly owned and supervised. With the increasing outward orientation of the economy and the financial sector liberalization, the authorities sought to modernize the legal framework governing the stock exchange activity in a market-oriented environment. Following the institutional settings in most major countries, the new law establishes three separate entities, a privately owned stock exchange, a clearing house (banque des titres), and an independent supervisory organization, the latter along the lines of the U.S. Securities and Exchange Commission. 4/ Banks are no longer allowed to act as stock

1/ TBs with a maturity of over one year purchased by banks at auctions can be converted into NTBs and sold to customers who then can trade them in the stock market.

2/ The total amount of negotiable treasury bonds issued was about TD 80 million in mid-1994, compared with 1.6 billion dinars of outstanding TBs.

3/ Tunisia has obtained technical assistance from the USAID to help improve the management of the domestic debt, including the settlement system.

4/ The Clearing House (banque des titres) existed since December 1993, but had not been fully operational. 
exchange brokers. The new legal framework is expected to allow for more efficiency in listing and trading, while providing appropriate safeguards against misuse. To avoid excess volatility, only proven sound companies are admitted to the bourse.

\section{Progress achieved}

In recent years, Tunisia has addressed the bulk of financial sector restrictions. It has achieved noteworthy results, in particular in the following areas:

Financial deepening: The abolition of interest rate controls and the introduction of longer-term bank instruments have led to the expected deepening of the market, as reflected in the increase in the ratio of $M 3 / M 1$ since 1990 (Chart 1). This point is also denonstrated by the growth and the increasing importance of longer term naturities in the treasury bill market (Chart 2). Finally, the number of investuent funds has increased dramatically (Chart 3 ).

Noninel and renl interest rntes: The 1987 reforms introduced more flexibility into the interest rate managenent, which has allowed the central bank to keep real interest rates positive. The central bank also raised preferential interest rates on various occasions, thus lowering, since 1990 , the implicit interest rate subsidy to currently around one percentage point. With the full liberalization of lending rates in 1994, remaining legal limits on interest rates only cover a small part of deposit rates. 1

Notwithstanding the far-reaching liberalization, observed nominal interest rate variability has remained low. This seems largely due to prevailing tacit understandings anong banks to keep deposit interest rates stable, while lending rates have continued to be informally linked to the money market rate (MM). 2) The last change in the TMY, which took place in November 1993, when the rate was lowered by 2 points from 10.8 percent to 8.8 percent, was largely reflected in lending rates. However, the renoval of the cap on average lending rates in June 1994, had no influence on the average level of lending rates, as it had not been binding.

As a result of the relatively stable nominal lending rates and falling inflation, real lending interest rates have remained positive. At about 7 percent, real rates in Tunisia are now about 1 percentage point above real rates in the United States. 3 /

1) Deposits of less than three months (maximum rate 2 percent), special savings accounts (TMM of the previous month - 2 points), accounts in convertible dinar (TMM - 2 points).

2) The TMM is largely influenced by the central bank's refinancing policy.

3/ Using the prime rate deflated by the consumer price index. 


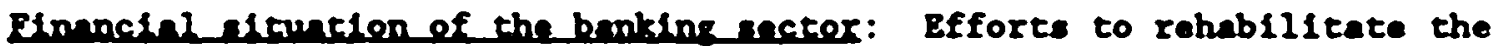
banking sector bave ylelded notable results. It appears from the prelinimary reaults of the audits that had bean carried out recently that a number of banks have already achieved, or are about to achieve, a capitalasset ratio of 5 percent. With respect to other banke, progress is being ande within the tine fram set by the authorities. Two large benke have successfully launched equity issues in the stock arket, while others contimu to bulld up capital based on retalned earnings. Helped by fiscal Incentives--the tax deductibility of provisions has been increased to 50 percent--banks have also reduced their vulnerabllity to riske with respect to outstanding loans.

Stock mekef: The increased dymalas of the stock narket is reflected In the nore than doubling of listinge and of the capitalization of the stock market (Chart 4). Also, the volus of eransactions rose sharply during the first three quarters of 1994, reflecting in part incressed recourse of private coupanies to bond financing as well as equity lsaus by a muber of consercial banks (Chart 5). The growth in stock values underscores the strong pent-up deand for equity in the face of a relatively weak supply. The new stock market law and an acceleration in the privatisation progran should facllitate an Increase in the offerings of stocks at the exchange. 
CHART 1

TUNISIA

FINANCIAL DEEPENING

(In percent; onnual overage)

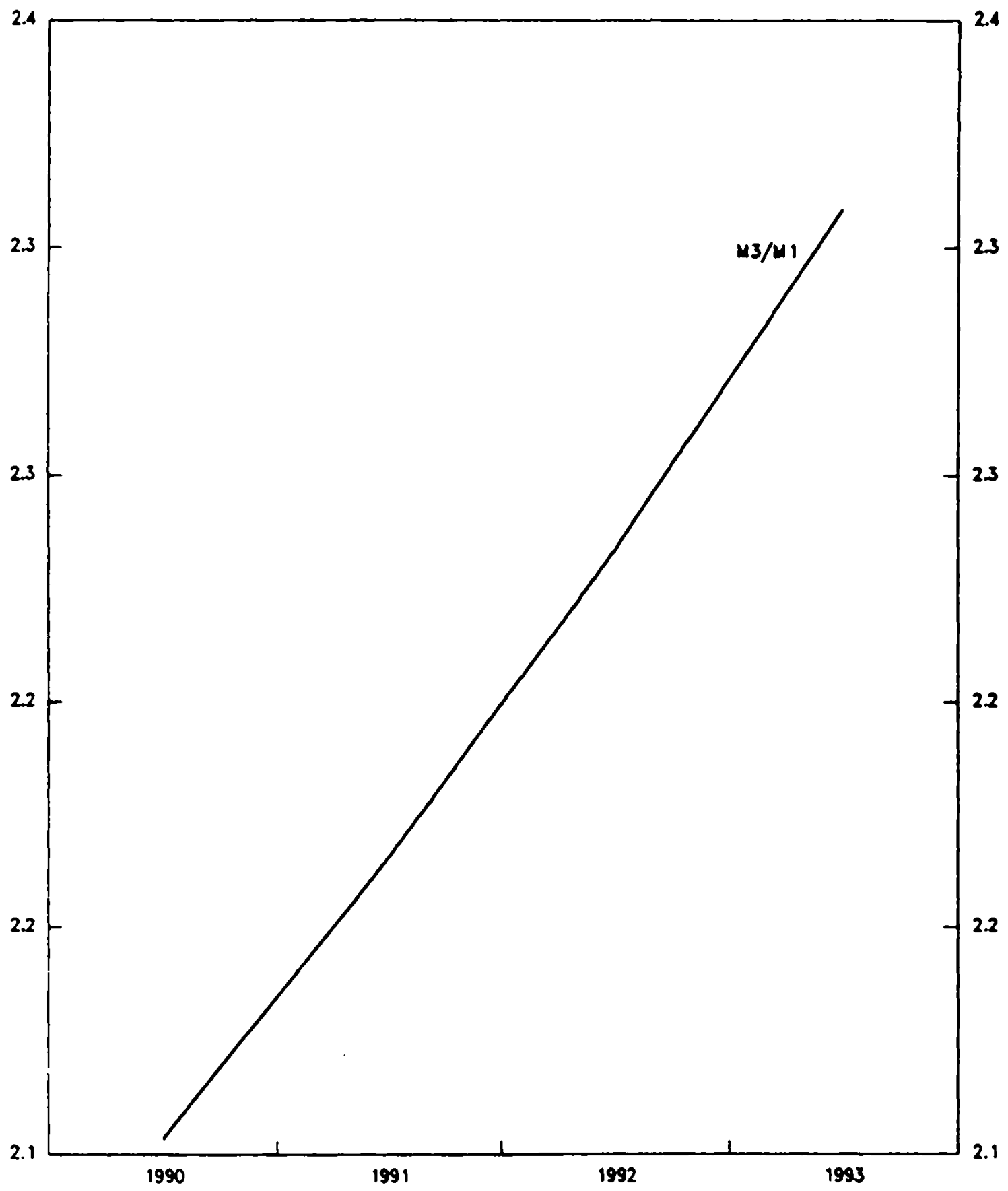

Source: Dato provided by the Tunision outhorlties.

(C) International Monetary Fund. Not for Redistribution 


\section{CHART 2 \\ TUNISIA \\ TREASURY BILLS OUTSTANDING}

(By moturity, in millions of dinors; end of period) $1 ;$

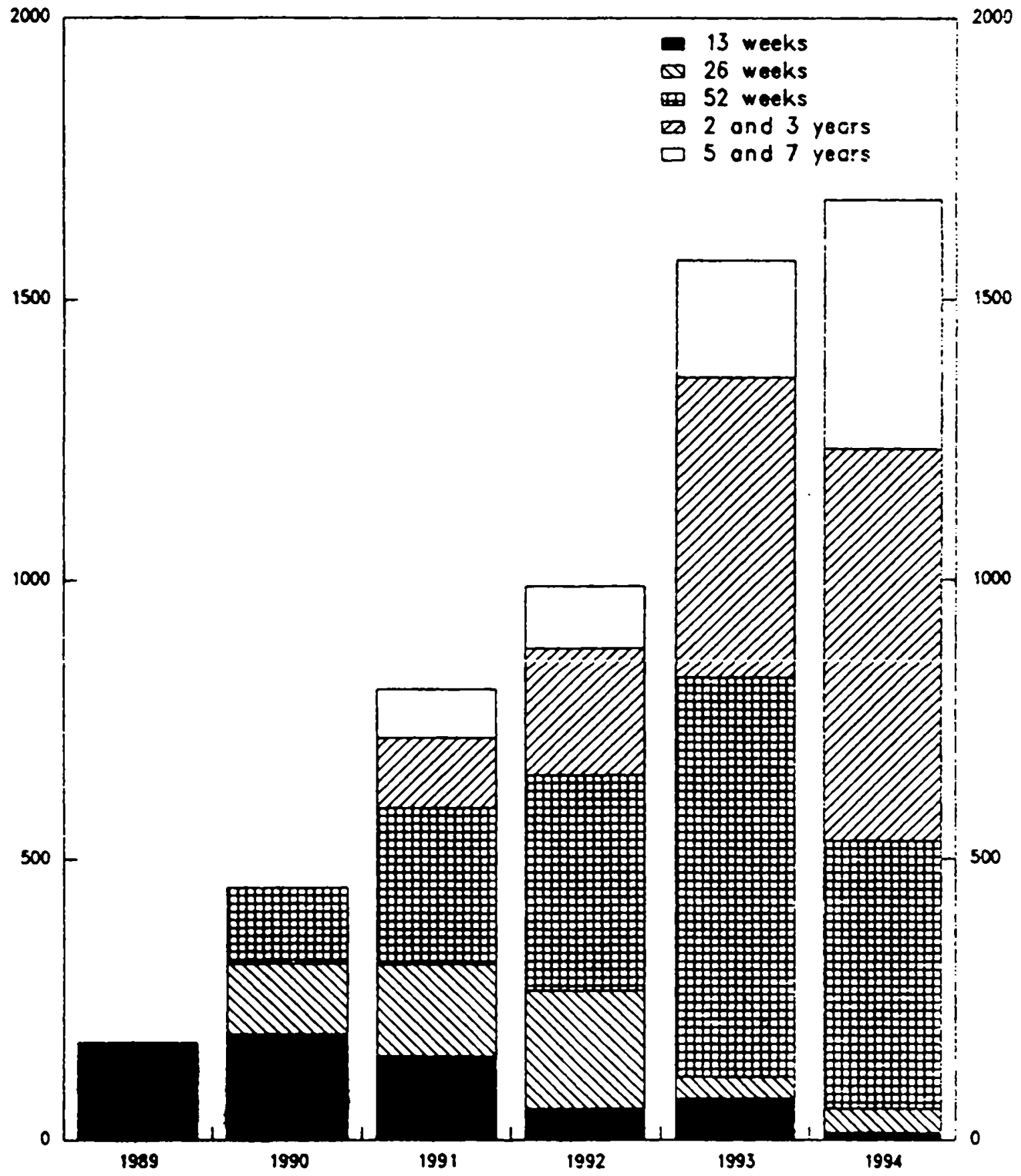

Source: Doto provided by the Tunision outhorities.

1/ Doto for 1994 ore for end-Soptember.

(C) International Monetary Fund. Not for Redistribution 
CHART 3

TUNISIA

NUMBER OF INVESTMENT FUNDS

(End of period) 1/

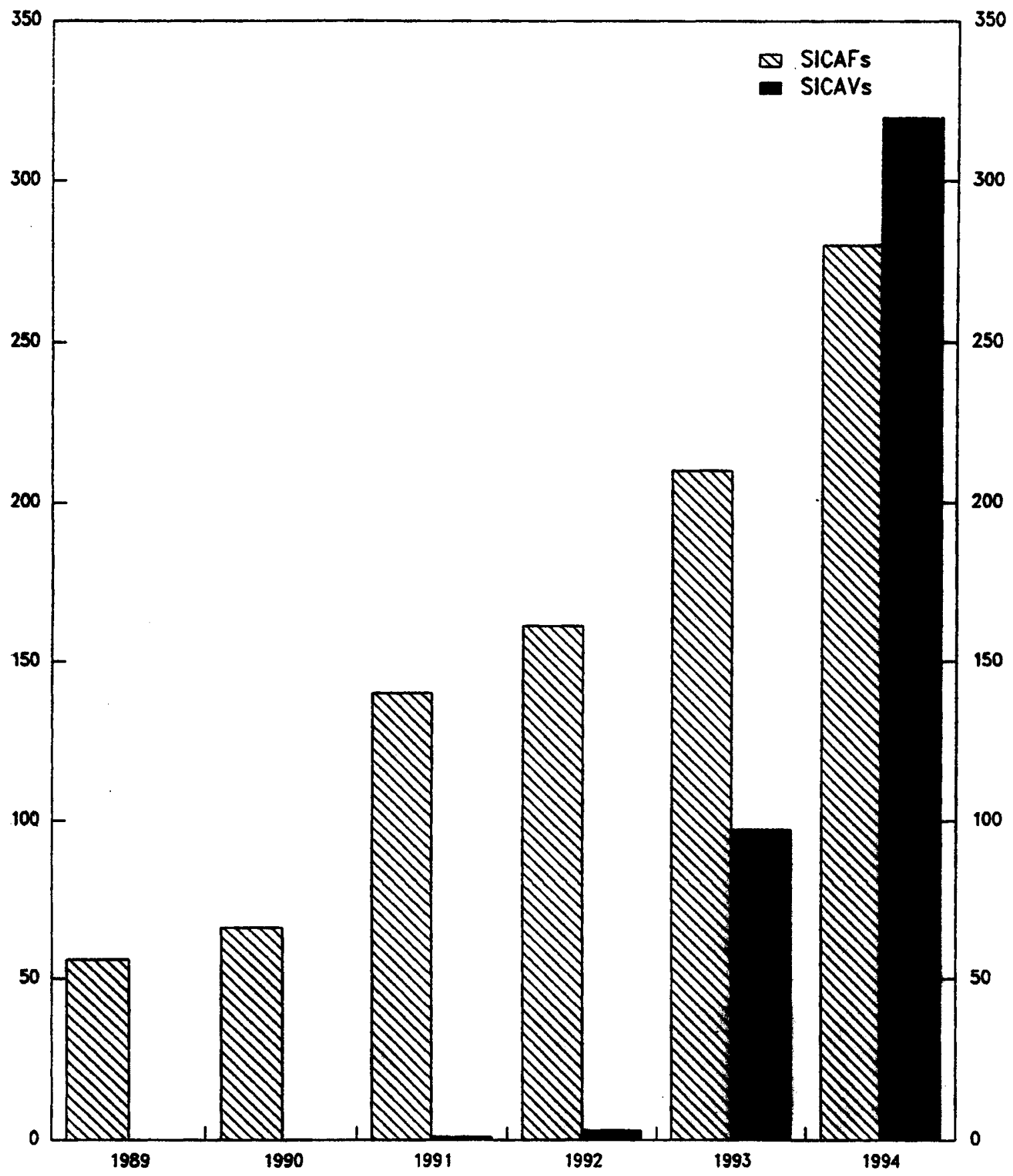

Source: Dato provided by the Tunision authorities.

1/ Dato for 1994 ore for end-September.

(CInternational Monetary Fund. Not for Redistribution 


\section{CHART 4 \\ TUNISIA \\ STOCK MARKET CAPITALIZATION \\ (In millions of dinars; end of period) $1 /$}

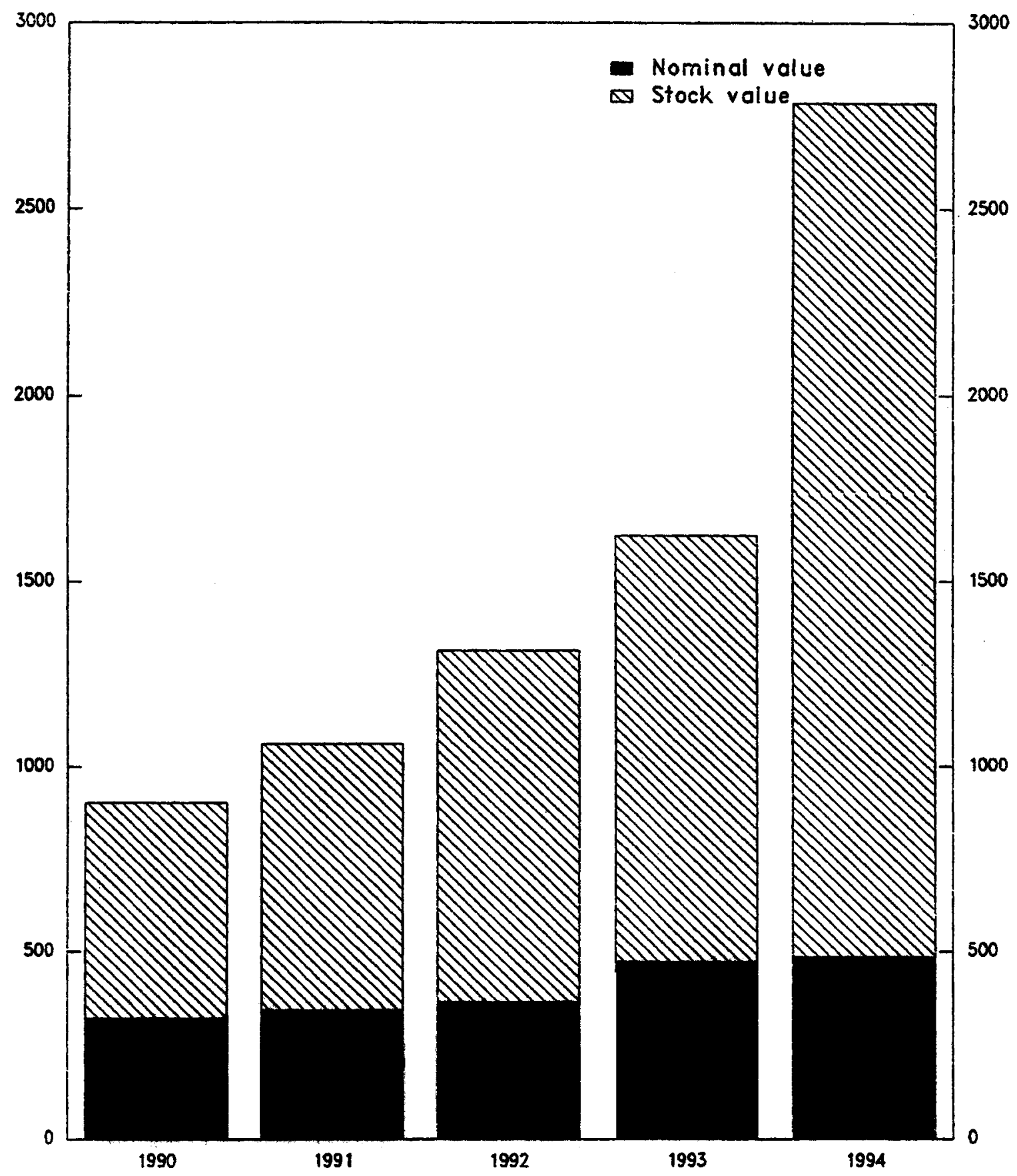

Source: Dato provided by the Tunision authorities.

1/ Data for 1994 are for end-September.

CInternational Monetary Fund. Not for Redistribution 


\section{CHART 5 \\ TUNISIA \\ STOCK MARKET DEVELOPMENT}

(Volume of transoctions; millions of dinors) 1/

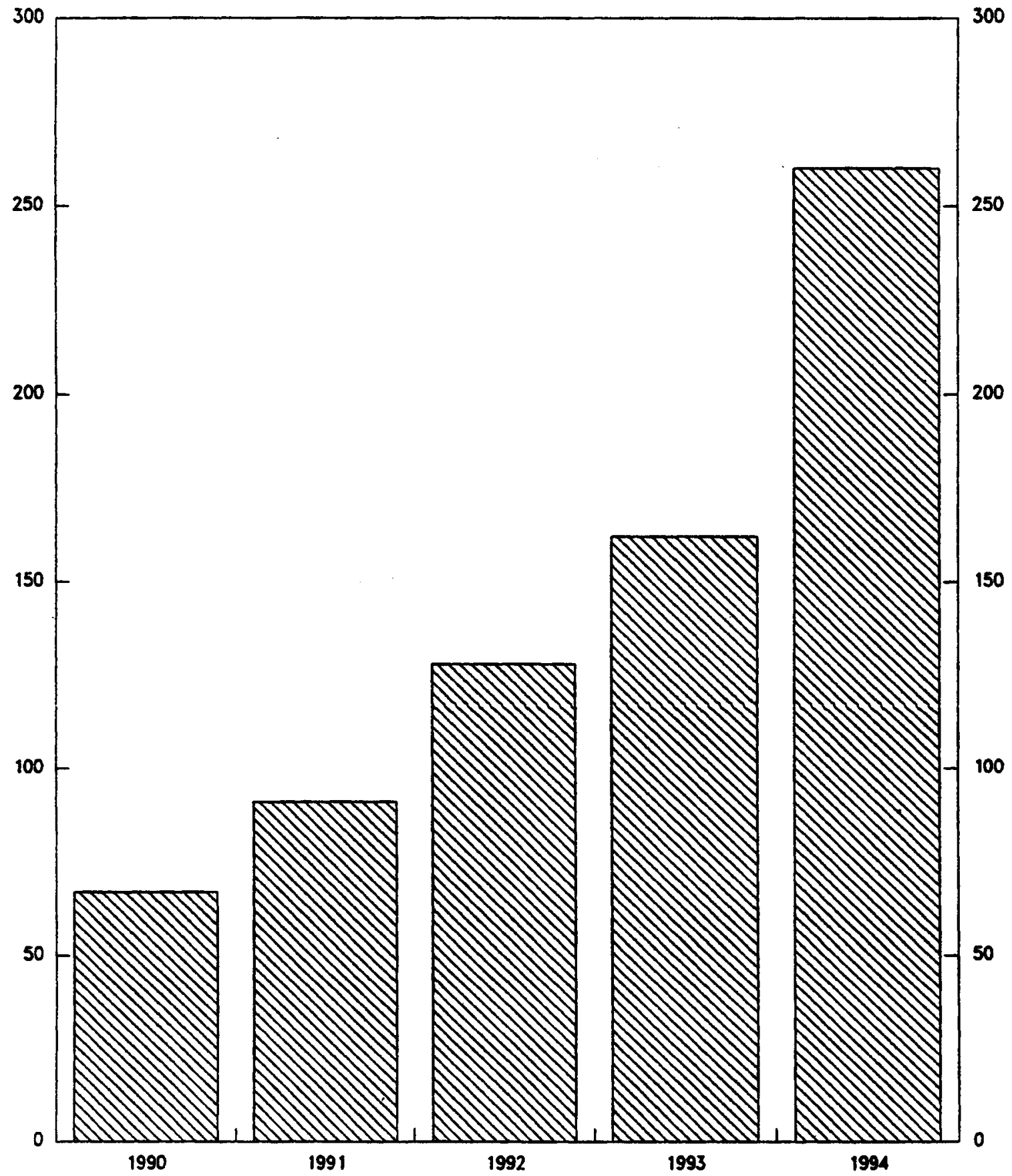

Source: Dato provided by the Tunision authorities.

1/ Dato for 1994 cover first 9 months.

CInternational Monetary Fund. Not for Redistribution 
Chronology of Major Financial Sector Liberalization Measures, 1987-94

\begin{tabular}{|c|c|c|}
\hline $\begin{array}{c}\text { Effective } \\
\text { Date }\end{array}$ & Legal Action & Measure \\
\hline January 1987 & BCT circular, Dec. 1986 & $\begin{array}{l}\text { Liberalization of interest rates } \\
\text { on term deposits of at least three } \\
\text { months. Rate on special savings } \\
\text { accounts set at the money market } \\
\text { rate (IMM) of the preceding month } \\
\text { minus two percentage points. } \\
\text { Deregulation of lending rates, } \\
\text { except those to priority sectors, } \\
\text { with retention of ceiling of } 3 \\
\text { percentage points above TMM. }\end{array}$ \\
\hline Dec. 1987 & BCT circular, Dec. 1987 & $\begin{array}{l}\text { Introduction of comprehensive bank } \\
\text { prudential regulations. }\end{array}$ \\
\hline January 1988 & BCT circular, Dec. 1987 & $\begin{array}{l}\text { Elimination of requirement for } \\
\text { prior approval of bank loans: } \\
\text { Rediscount operations restricted } \\
\text { to loans to priority sectors. } \\
\text { streamlining of lending to } \\
\text { priority sectors. }\end{array}$ \\
\hline January 1988 & BCT circular, Dec. 1987 & $\begin{array}{l}\text { Opening of money market to } \\
\text { interbank transactions, and to } \\
\text { operations in certificates of } \\
\text { deposit and commercial paper. } \\
\text { Establishing of Central Bank } \\
\text { intervention in money market as } \\
\text { main policy instrument. }\end{array}$ \\
\hline August 1988 & Law, August 1988 & $\begin{array}{l}\text { Reform of closed-end and open-end } \\
\text { investment funds, which were } \\
\text { granted fiscal and financial } \\
\text { incentives. }\end{array}$ \\
\hline March 1989 & $\begin{array}{l}\text { Avis de change of the } \\
\text { Minister of Finance, } \\
\text { February } 1989 \text { and BCT } \\
\text { circular, March } 1989\end{array}$ & $\begin{array}{l}\text { Creation of system of foreign } \\
\text { currency options. }\end{array}$ \\
\hline March 1989 & Law, March 1989 & $\begin{array}{l}\text { Strengthening of Stock Exchange } \\
\text { operations. }\end{array}$ \\
\hline
\end{tabular}




\begin{tabular}{|c|c|c|}
\hline$\frac{\text { Effective }}{\text { Date }}$ & Legal Action & Measure \\
\hline May 1989 & BCT circular, May 1989 & $\begin{array}{l}\text { Broadening of modalities for } \\
\text { Central Bank intervention in money } \\
\text { market and liberalization of } \\
\text { conditions for issue of commercial } \\
\text { paper. }\end{array}$ \\
\hline May 1989 & BCT circular, May 1989 & $\begin{array}{l}\text { Creation of money market in } \\
\text { foreign currency. }\end{array}$ \\
\hline June 1989 & BCT circular, May 1989 & $\begin{array}{l}\text { Reactivation of reserve } \\
\text { requirenent while nodifying it } \\
\text { into a more prudential tool and a } \\
\text { possible monetary policy } \\
\text { instrument. }\end{array}$ \\
\hline August 1989 & $\begin{array}{l}\text { Avis de change of } \\
\text { Minister of Finance, } \\
\text { July } 1989 \text { and BCT } \\
\text { circular, August } 1989 \\
\end{array}$ & $\begin{array}{l}\text { widening of scope of forward } \\
\text { exchange cover. }\end{array}$ \\
\hline Sept. 1989 & $\begin{array}{l}\text { Arrété of Minister of } \\
\text { Finance and BCT } \\
\text { circular, Sept. } 1989\end{array}$ & $\begin{array}{l}\text { Introduction of Treasury Bills } \\
\text { with terms up to } 52 \text { weeks. }\end{array}$ \\
\hline March 1991 & $\begin{array}{l}\text { Note to banks, March } \\
1991\end{array}$ & $\begin{array}{l}\text { Relaxation of mandatory bank } \\
\text { holdings of Treasury debt } \\
\text { instruments by allowing placenent } \\
\text { with public. }\end{array}$ \\
\hline April 1991 & $\begin{array}{l}\text { BCT circular, April } \\
1991\end{array}$ & $\begin{array}{l}\text { Further liberalization of } \\
\text { conditions for issuance of } \\
\text { commercial paper. Minimum term } \\
\text { for certificates of deposits } \\
\text { increased from } 10 \text { to } 90 \text { days. }\end{array}$ \\
\hline Nov. 1991 & $\begin{array}{l}\text { Arrêtés of the Minister } \\
\text { of Finance, June and } \\
\text { September 1991, and BCT } \\
\text { circular, November } 1991 \\
\end{array}$ & $\begin{array}{l}\text { Introduction of treasury bills } \\
\text { with terms exceeding one year. }\end{array}$ \\
\hline January 1992 & BCT circular, Dec. 1991 & $\begin{array}{l}\text { Further liberalization of lending } \\
\text { rates. Ceiling on individual loan } \\
\text { rates replaced by ceiling on } \\
\text { average lending rates per bank set } \\
\text { at TMM plus } 3 \text { percentage points. }\end{array}$ \\
\hline
\end{tabular}




\begin{tabular}{|c|c|c|}
\hline $\begin{array}{c}\text { Effective } \\
\text { Pate }\end{array}$ & Legen Action & Mensure \\
\hline Jamuary 1992 & BCT circular, Dec. 1991 & $\begin{array}{l}\text { Strengthening of prudential } \\
\text { regulations, mainly through } \\
\text { setting exposure linits. loan } \\
\text { classifications and loan loss } \\
\text { provisions and capital asset } \\
\text { reguirements. }\end{array}$ \\
\hline Jamuary 1992 & $\begin{array}{l}1992 \text { Finance Law, } \\
\text { Deceaber } 1991\end{array}$ & $\begin{array}{l}\text { Harmonization of tax treatant of } \\
\text { financial instruments. Increase } \\
\text { in tax exaption for bank. } \\
\text { provisions. }\end{array}$ \\
\hline June 1992 & $\begin{array}{l}\text { Avls de change, } \\
\text { Minlster of Finance, } \\
\text { Sept. } 1991 \text { and BCT } \\
\text { circular, June } 1992 \\
\end{array}$ & $\begin{array}{l}\text { Extension of operations in the } \\
\text { woney market in foreign exchange. }\end{array}$ \\
\hline June 1992 & BCT circular, June 1992 & $\begin{array}{l}\text { Reduction of scope of credits at } \\
\text { preferential interest retes. }\end{array}$ \\
\hline Nov. 1992 & Law, Nov. 1992 & $\begin{array}{l}\text { Creation of now financial } \\
\text { instruments, including invescoent } \\
\text { trusts, priority shares and equity } \\
\text { loans. }\end{array}$ \\
\hline Nov. 1992 & Law, Nov. 1992 & $\begin{array}{l}\text { Liberalization of operations and } \\
\text { increase in ainimu required } \\
\text { capteal for closed-end investment } \\
\text { funds. }\end{array}$ \\
\hline January 1993 & $\begin{array}{l}1993 \text { Finance Lew, } \\
\text { Decesber } 1992\end{array}$ & $\begin{array}{l}\text { Further increase in tax exemption } \\
\text { for bank provisions. Introduction } \\
\text { of tax exantion for capital gains } \\
\text { of banks. }\end{array}$ \\
\hline Jamuary 1993 & $\begin{array}{l}\text { BCT notification to } \\
\text { banks, Jan. } 1993 \text {. } \\
\text { Formailized by Law, May } \\
1993 \text {, Decree, August } \\
1993 \text { and BCT circulars, } \\
\text { Septenber, October and } \\
\text { December 1993, and May } \\
\text { and September } 1994\end{array}$ & $\begin{array}{l}\text { Establishment of current account } \\
\text { convertibility. }\end{array}$ \\
\hline January 1993 & $\begin{array}{l}\text { BCT notification to } \\
\text { banks, Jan. 1993. } \\
\text { Foralized by Decree, } \\
\text { August 1993, and BCT } \\
\text { circular, October } 1993\end{array}$ & $\begin{array}{l}\text { Partial liberalization of foreign } \\
\text { borrowing by resident enterprises } \\
\text { and financial institutions. }\end{array}$ \\
\hline
\end{tabular}




\begin{tabular}{|c|c|c|}
\hline $\begin{array}{c}\text { Effective } \\
\text { Pate }\end{array}$ & Lerel Action & Mensure \\
\hline May 1993 & Law, May 1993 & $\begin{array}{l}\text { Extension of right to repatriate } \\
\text { proceeds of liquidation of capital } \\
\text { invested on the basis of foreign } \\
\text { exchange transfer extended to all } \\
\text { investeents by nonresidents. }\end{array}$ \\
\hline Januery 1994 & $\begin{array}{l}1994 \text { Finance Law. } \\
\text { December } 1993\end{array}$ & $\begin{array}{l}\text { Tax exemption for bank provisions } \\
\text { further increased. }\end{array}$ \\
\hline Feb. 1994 & Law. Fob. 1994 & $\begin{array}{l}\text { New banking law: Sets legal. } \\
\text { framework for a more market- } \\
\text { oriented banking systen by opening } \\
\text { new areas of business; aboliahes } \\
\text { the need for banks to hold public } \\
\text { debt and institutes beter and } \\
\text { wore comprehensive prudential } \\
\text { requireants; creates the banque } \\
\text { d'affaires. }\end{array}$ \\
\hline March 1994 & $\begin{array}{l}\text { BCT circular, March } \\
1994\end{array}$ & $\begin{array}{l}\text { Creation of an Interbank foreign } \\
\text { exchange waket. The BCT } \\
\text { continues to announce a central } \\
\text { rate; banks are allowed to have } \\
\text { open foreign exchange positions } \\
\text { within prudential liaits. }\end{array}$ \\
\hline June 1994 & $\begin{array}{l}\text { Avis de change of } \\
\text { Minister of Finance, } \\
\text { Dec. } 1993 \text { and BCT } \\
\text { circular, Jume } 1994 \\
\end{array}$ & $\begin{array}{l}\text { Liberalization of outwerd foreign } \\
\text { direct investent for exporting } \\
\text { enterprises in linited seounts. }\end{array}$ \\
\hline June 1994 & BCT c1rcular, June 1994 & $\begin{array}{l}\text { Complete liberalization of lending } \\
\text { rates for nompriority sectors. }\end{array}$ \\
\hline Nov. 1994 & Law, Nov. 1994 & $\begin{array}{l}\text { Reform of the stock market: } \\
\text { Privatizes the stock market; } \\
\text { creates an independent supervisory } \\
\text { body. Also legalizes the already } \\
\text { (yet inoperative) settlement body. }\end{array}$ \\
\hline
\end{tabular}


Table 1. Tunitin: Real ODP Growth by Sector, 1990-94

(in peroent)

\begin{tabular}{|c|c|c|c|c|c|}
\hline & 1990 & 1991 & 1992 & 1993 & $\begin{array}{l}\text { Prel. } \\
\frac{\text { Ert. }}{1994}\end{array}$ \\
\hline $\begin{array}{l}\text { Agriculture and fithing } \\
\text { Mining } \\
\text { Hydrocurbons, electricity, and water }\end{array}$ & $\begin{array}{r}35.2 \\
-17.9 \\
-6.1\end{array}$ & $\begin{array}{r}14.5 \\
0.1 \\
11.4\end{array}$ & $\begin{array}{r}5.4 \\
-3.3 \\
1.4\end{array}$ & $\begin{array}{r}-5.9 \\
-13.3 \\
-6.6\end{array}$ & $\begin{array}{r}-6.9 \\
14.2 \\
-0.7\end{array}$ \\
\hline 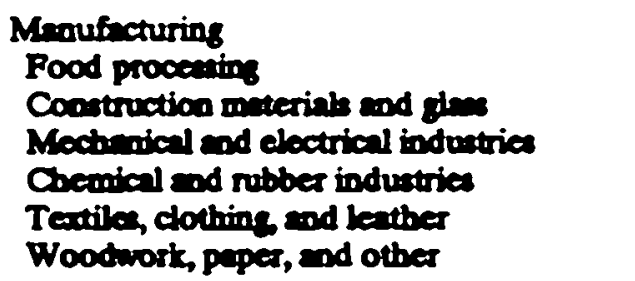 & $\begin{array}{r}1.8 \\
-9.9 \\
-9.5 \\
2.8 \\
-18.5 \\
23.0 \\
9.9\end{array}$ & $\begin{array}{l}4.9 \\
9.1 \\
1.5 \\
3.5 \\
2.9 \\
4.3 \\
4.8\end{array}$ & $\begin{array}{r}7.6 \\
11.6 \\
4.3 \\
4.5 \\
4.8 \\
8.2 \\
7.3\end{array}$ & $\begin{array}{r}2.7 \\
-7.9 \\
5.4 \\
3.7 \\
4.2 \\
6.6 \\
8.5\end{array}$ & $\begin{array}{r}7.7 \\
10.0 \\
3.2 \\
5.4 \\
7.8 \\
9.0 \\
7.6\end{array}$ \\
\hline Mroufacturins, exctuding food procesaing & 5.7 & 3.7 & 6.4 & 6.0 & 7.1 \\
\hline Construction end public worts & 14.0 & -0.5 & 6.3 & 8.5 & 8.0 \\
\hline $\begin{array}{l}\text { Services } \\
\text { Trimport and telecomminications } \\
\text { Lod gine food, ad beverages } \\
\text { Commence and other services } \\
\text { Nonmertet services }\end{array}$ & $\begin{array}{r}4.8 \\
7.2 \\
-2.1 \\
7.2 \\
3.2\end{array}$ & $\begin{array}{r}-0.9 \\
-1.3 \\
-25.0 \\
2.3 \\
4.3\end{array}$ & $\begin{array}{r}10.8 \\
12.6 \\
44.8 \\
7.7 \\
4.4\end{array}$ & $\begin{array}{l}6.0 \\
6.7 \\
9.1 \\
6.1 \\
4.3\end{array}$ & $\begin{array}{r}7.1 \\
7.7 \\
14.0 \\
7.0 \\
4.2\end{array}$ \\
\hline Indirect tures mimus aubridies & 8.8 & 2.1 & 8.5 & 4.2 & 6.1 \\
\hline GDP (at market prices) & 7.8 & 3.9 & 8.0 & 21 & 4.4 \\
\hline
\end{tabular}

Source: Ministry of Plenning and Regional Development. 
Table 2. Tunisia: Suppty and Use of Resources - Real Grouth Rates, 1990-94

(In percent)

\begin{tabular}{|c|c|c|c|c|c|}
\hline & 1990 & 1991 & 1992 & 1993 & $\begin{array}{l}\text { Prel. } \\
\text { Est. } \\
1994\end{array}$ \\
\hline Consumption & 6.0 & 1.9 & 5.7 & 3.1 & 4.3 \\
\hline $\begin{array}{l}\text { Private soctor } \underline{y} \\
\text { Central Government }\end{array}$ & $\begin{array}{l}6.5 \\
32\end{array}$ & $\begin{array}{l}0.7 \\
9.3\end{array}$ & $\begin{array}{l}5.9 \\
4.5\end{array}$ & $\begin{array}{l}28 \\
4.5\end{array}$ & $\begin{array}{l}4.5 \\
2.7\end{array}$ \\
\hline $\begin{array}{l}\text { Gross frued capital } \\
\text { formation }\end{array}$ & 13.5 & 3.7 & 18.0 & 8.0 & 6.8 \\
\hline Domestic demand & 9.0 & 2.0 & 9.1 & 2.4 & 4.5 \\
\hline $\begin{array}{l}\text { Exports of goods and } \\
\text { nonfactor services }\end{array}$ & 26 & -3.6 & 10.7 & 25 & 11.0 \\
\hline $\begin{array}{l}\text { Imports of goods and } \\
\text { nonfactor services }\end{array}$ & 5.8 & -6.8 & 13.2 & 3.0 & 10.3 \\
\hline GDP at market prices & 7.8 & 3.9 & 8.0 & 2.1 & 4.4 \\
\hline
\end{tabular}

Source: Ministry of Planning and Regional Development.

1/ Includes all economic agents cocept the Central Government. 
Table 3. Tunisia: Sectaral Diveribution of ODP, 1900-94

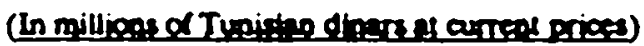

\begin{tabular}{|c|c|c|c|c|c|c|}
\hline & 1909 & 1990 & 1991 & 1992 & 1993 & $\begin{array}{l}\text { Prel } \\
\frac{\text { Ex }}{1994}\end{array}$ \\
\hline $\begin{array}{l}\text { Aproukure and fubing } \\
\text { Mining } \\
\text { Hydrocarboes, elecaricity. } \\
\text { ad weler }\end{array}$ & $\begin{array}{r}1261 \\
139 \\
843\end{array}$ & $\begin{array}{r}1,763 \\
100\end{array}$ & $\begin{array}{r}2.134 \\
97\end{array}$ & $\begin{array}{r}2371 \\
85\end{array}$ & $\frac{2,205}{78}$ & $\begin{array}{r}\frac{220}{89} \\
904\end{array}$ \\
\hline 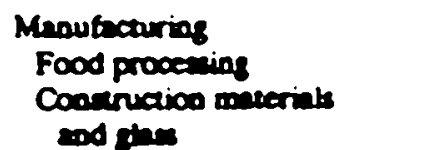 & $\begin{array}{r}1,655 \\
357 \\
211\end{array}$ & $\begin{array}{r}1,796 \\
394\end{array}$ & $\begin{array}{r}2011 \\
450\end{array}$ & $\begin{array}{r}2278 \\
528\end{array}$ & $\begin{array}{r}2,470 \\
\operatorname{sen}\end{array}$ & $\begin{array}{r}2786 \\
\text { S5S }\end{array}$ \\
\hline 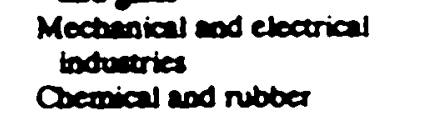 & 205 & 2803 & 316 & 305 & 375 & 419 \\
\hline indinatios & 214 & 143 & 155 & 165 & 178 & 200 \\
\hline $\begin{array}{l}\text { leather } \\
\text { Woodmort, paper, and ocher }\end{array}$ & $\begin{array}{l}137 \\
210\end{array}$ & $\begin{array}{l}535 \\
238\end{array}$ & $\begin{array}{l}598 \\
200\end{array}$ & $\begin{array}{l}600 \\
300\end{array}$ & 353 & $\begin{array}{l}916 \\
400\end{array}$ \\
\hline $\begin{array}{l}\text { Manufiauriog. exchuding food } \\
\text { procesting }\end{array}$ & 1298 & 1,402 & 1561 & 1.750 & 1,968 & 2230 \\
\hline Consuruction ans public worts & 359 & $\infty$ & 479 & 520 & 506 & 671 \\
\hline $\begin{array}{l}\text { Services } \\
\text { Tranepon and }\end{array}$ & 4,194 & 4613 & 4,558 & 5812 & 6,514 & 7,330 \\
\hline 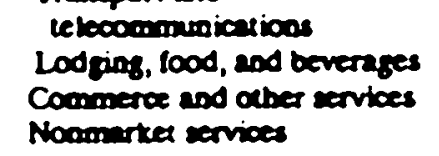 & $\begin{array}{r}604 \\
527 \\
1,604 \\
1,308\end{array}$ & $\begin{array}{l}780 \\
552 \\
1,899 \\
1,411\end{array}$ & $\begin{array}{r}803 \\
2009 \\
1618\end{array}$ & $\begin{array}{r}1,008 \\
645 \\
2,350 \\
1,009\end{array}$ & $\begin{array}{r}1,140 \\
764 \\
2027 \\
1984\end{array}$ & $\begin{array}{r}1204 \\
913 \\
2,952 \\
2,173\end{array}$ \\
\hline GDP a tactor $\infty$ & 8,451 & 9,509 & 10568 & 12,002 & 12831 & 14,004 \\
\hline Indirea taves minus subsidies & $1,0: 0$ & 1229 & 1,430 & 1,733 & 1857 & 2,130 \\
\hline GDP at martet prices & 9,531 & 10,798 & 11,997 & 13,754 & 14,688 & 16,134 \\
\hline
\end{tabular}

Source: Minisery of Planning and Regional Developmeat. 
Table 4. Tuabia: Supply and Use of Reaurcen, 1989-94

\begin{tabular}{|c|c|c|c|c|c|c|}
\hline & 1989 & 1990 & 1991 & 1992 & 1993 & $\frac{\text { Ets. }}{1994}$ \\
\hline & \multicolumn{6}{|c|}{ 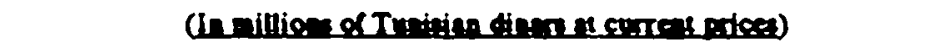 } \\
\hline Comuenption & 7,677 & 8.635 & 9,434 & 10,522 & 11,355 & 12,481 \\
\hline $\begin{array}{l}\text { Private sector } 1 \\
\text { Ceatral Government }\end{array}$ & $\begin{array}{l}6,520 \\
1,157\end{array}$ & $\begin{array}{l}7,390 \\
1,245\end{array}$ & $\begin{array}{l}7,990 \\
1,444\end{array}$ & $\begin{array}{l}8,932 \\
1,590\end{array}$ & $\begin{array}{l}9,586 \\
1,769\end{array}$ & $\begin{array}{r}10,538 \\
1,943\end{array}$ \\
\hline \multicolumn{6}{|l|}{ Grows fixed capital } & 4,658 \\
\hline $\begin{array}{l}\text { Privete cector } y \\
\text { Ceatral Governament }\end{array}$ & $\begin{array}{r}1,754 \\
403\end{array}$ & $\begin{array}{r}2.137 \\
498\end{array}$ & $\begin{array}{r}2.215 \\
697\end{array}$ & $\begin{array}{r}3,075 \\
560\end{array}$ & $\begin{array}{r}3,515 \\
653\end{array}$ & $\begin{array}{r}3,914 \\
744\end{array}$ \\
\hline Chaseses in stocks & 101 & 242 & 231 & 496 & 140 & 82 \\
\hline Domeatic demand & 9,935 & 11,511 & $12,5 n$ & 14.653 & 15,603 & 17,221 \\
\hline Net aports of goods asd & -404 & -714 & -580 & -899 & -974 & -1.087 \\
\hline $\begin{array}{l}\text { Exporis of goodh and } \\
\text { Donfect or senvices }\end{array}$ & 4,317 & 4,662 & 4.802 & 5,357 & $\$, 912$ & 6,800 \\
\hline $\begin{array}{l}\text { Imporis of goods and } \\
\text { nosfactor services }\end{array}$ & 4,722 & 5,376 & 5,382 & 6,256 & 6,887 & 7,887 \\
\hline GDP \& nartet prices & 9,531 & 10,793 & $11, \operatorname{sen}$ & 13,754 & 14,608 & 16,134 \\
\hline $\begin{array}{l}\text { Net iscome from } \\
\text { abrosd }\end{array}$ & -390 & -359 & -500 & -519 & -762 & -689 \\
\hline GNP & 9,142 & 10,438 & 11,498 & 13,236 & 13,926 & 15,445 \\
\hline Net curreat transfen from abroad & 450 & sos & 515 & 501 & 574 & 656 \\
\hline \multirow[t]{2}{*}{ Grow national disposable iscoene } & 9,591 & 10,943 & 12,013 & 13,736 & 14,500 & 26,101 \\
\hline & \multicolumn{6}{|c|}{ (In percent o( GDP) } \\
\hline \multicolumn{7}{|l|}{ Memorandum items: } \\
\hline Grone ioveatsess & 23.7 & 26.6 & 26.2 & 30.0 & 29.3 & 29.4 \\
\hline Gros doseatic suning & 19.4 & 20.0 & 21.4 & 23.5 & 22.7 & 22.6 \\
\hline Groes sutional eoviog & 20.1 & 21.4 & 21.5 & 23.4 & 21.4 & 224 \\
\hline Soviog - invealment gap & -3.6 & -5.3 & -4.7 & -6.7 & -7.9 & -6.9 \\
\hline Privare sector $y$ & -2.2 & -4.8 & -1.6 & -6.6 & -7.7 & -6.5 \\
\hline Cencral govetnment & -1.4 & -0.5 & -3.1 & -0.1 & -0.3 & -0.4 \\
\hline
\end{tabular}

Source: Ministry of Planning and Regional Deve'npment.

y Includes all exonomic asents except the Central Government 
Table 5. Tuniaia: Production of Major Africultural Crops, 1989-94

(In thourands of metric tops)

\begin{tabular}{|c|c|c|c|c|c|c|}
\hline & 1989 & 1990 & 1991 & 1992 & 1993 & 1994 \\
\hline $\begin{array}{l}\text { Cereals } \\
\text { Hard wbeat } \\
\text { Soft wbeat } \\
\text { Barley }\end{array}$ & $\begin{array}{r}635 \\
333 \\
87 \\
215\end{array}$ & $\begin{array}{r}1,633 \\
897 \\
225 \\
511\end{array}$ & $\begin{array}{r}2,551 \\
1,424 \\
363 \\
765\end{array}$ & $\begin{array}{r}2,195 \\
1,323 \\
261 \\
611\end{array}$ & $\begin{array}{r}1,914 \\
1,134 \\
279 \\
501\end{array}$ & $\begin{array}{r}654 \\
436 \\
66 \\
152\end{array}$ \\
\hline $\begin{array}{l}\text { Fruits and vegetables } \\
\text { Citrus truit } \\
\text { Dates } \\
\text { Tomatoes } \\
\text { Red peppers } \\
\text { Potatoes } \\
\text { Melons and watermelons } \\
\text { Almonds } \\
\text { Table grapes }\end{array}$ & $\begin{array}{r}260 \\
75 \\
440 \\
110 \\
180 \\
250 \\
35 \\
50\end{array}$ & $\begin{array}{r}237 \\
81 \\
530 \\
175 \\
217 \\
450 \\
52 \\
40\end{array}$ & $\begin{array}{r}226 \\
75 \\
580 \\
180 \\
220 \\
450 \\
40 \\
60\end{array}$ & $\begin{array}{r}185 \\
75 \\
550 \\
190 \\
218 \\
380 \\
45 \\
60\end{array}$ & $\begin{array}{r}281 \\
86 \\
420 \\
180 \\
200 \\
330 \\
47 \\
60\end{array}$ & $\begin{array}{r}210 \\
74 \\
480 \\
165 \\
210 \\
375 \\
52 \\
55\end{array}$ \\
\hline Olives for oil & 270 & 650 & 825 & 1,325 & 675 & 1,050 \\
\hline $\begin{array}{l}\text { Otber } \\
\text { Meat } \\
\text { Sugar beets } \\
\text { Mill } \\
\text { Firh }\end{array}$ & $\begin{array}{r}130 \\
230 \\
380 \\
95\end{array}$ & $\begin{array}{r}132 \\
289 \\
400 \\
88\end{array}$ & $\begin{array}{r}136 \\
210 \\
419 \\
88\end{array}$ & $\begin{array}{r}141 \\
291 \\
49 \\
89\end{array}$ & $\begin{array}{r}151 \\
245 \\
486 \\
83\end{array}$ & $\begin{array}{r}153 \\
232 \\
500 \\
84\end{array}$ \\
\hline
\end{tabular}

Source: Minintry of Arriculture. 
Table 6. Tunisia: Supply and Use of Cereals, 1989/90-1993/94 1/

(In thousands of tons)

\begin{tabular}{|c|c|c|c|c|c|}
\hline & $1989 / 90$ & 1990/91 & 1991/92 & 1992/93 & 1993/94 \\
\hline \multicolumn{6}{|l|}{ Hard wheat } \\
\hline Production & 897 & 1,424 & 1,323 & 1,134 & 436 \\
\hline $\begin{array}{l}\text { Commercialization } 2 \text { f } \\
\text { (in percent of previous year) }\end{array}$ & $\begin{array}{r}288 \\
81\end{array}$ & $\begin{array}{r}484 \\
86\end{array}$ & $\begin{array}{r}772 \\
54\end{array}$ & $\begin{array}{r}862 \\
65\end{array}$ & $\begin{array}{r}786 \\
69\end{array}$ \\
\hline Imports & 100 & 128 & -- & -- & - \\
\hline Producer price (D/t) $3 /$ & 245 & 245 & 260 & 260 & 260 \\
\hline Real producer price index 4 & 130 & 120 & 121 & 115 & 110 \\
\hline \multicolumn{6}{|l|}{ Soft wheat } \\
\hline Production & 225 & 363 & 261 & 279 & 66 \\
\hline $\begin{array}{l}\text { Commercialization } 2 \mathrm{f} \\
\text { (in percent of previous year) }\end{array}$ & $\begin{array}{l}68 \\
73\end{array}$ & $\begin{array}{r}132 \\
78\end{array}$ & $\begin{array}{r}228 \\
59\end{array}$ & $\begin{array}{r}224 \\
86\end{array}$ & $\begin{array}{r}201 \\
72\end{array}$ \\
\hline Imports & 622 & 488 & 623 & 550 & 752 \\
\hline Producer price (D/t) $3 /$ & 209 & 209 & 225 & 225 & 225 \\
\hline Real producer price index 4/ & 123 & 114 & 117 & 110 & 105 \\
\hline \multicolumn{6}{|l|}{ Barley } \\
\hline Production & 478 & 721 & 570 & 478 & 145 \\
\hline Commercialization 2 & 29 & 143 & 292 & 188 & 87 \\
\hline (in percent of previous year) & 11 & 15 & 30 & 34 & 18 \\
\hline Imports & 126 & -- & -- & -- & -- \\
\hline Producer price (D/t) $3 /$ & 150 & 150 & 150 & 150 & 150 \\
\hline Real producer price index 4 & 115 & 107 & 101 & 96 & 92 \\
\hline
\end{tabular}

Source: Tunisian authorities.

1/ By crop year (July/June).

2) Commercialization of the previous year's crop.

3) D/t, dinars per ton.

4) Index $1980=100$. Nominal producer prices deflated by the consumer price index. 
Table 7. Tunisia: Producer Prices of Principal Agricultural Commodities, 1989-94 1/

(In Tunisian dinars per ton)

\begin{tabular}{lrrrrrr}
\hline & 1989 & 1990 & 1991 & 1992 & 1993 & 1994 \\
\hline Cereals & & & & & & \\
$\quad$ Hard wheat & 225 & 245 & 245 & 260 & 260 & 260 \\
$\begin{array}{l}\text { Soft wheat } \\
\text { Barley }\end{array}$ & 199 & 209 & 209 & 225 & 225 & 225 \\
Sugar beets & 145 & 150 & 150 & 150 & 150 & 150 \\
Olives for oil & 25 & 30 & 32 & 35 & 39 & 39 \\
$\quad$ High grade & 1,500 & 1,710 & 1,750 & 1,750 & 1,750 & 1,315 \\
$\quad$ Low grade & 1,260 & 1,360 & 1,390 & 1,390 & 1,390 & 1,055 \\
Tomatoes & 70 & 70 & 70 & 70 & 70 & 90 \\
\hline
\end{tabular}

Source: Tunisian authorities.

1/ For crop year (July/June). 
Table 8. Tuniaia: Energy Production and Cocaumption, 19.9-94

\begin{tabular}{|c|c|c|c|c|c|c|}
\hline & 1989 & 1990 & 1991 & 1992 & 1993 & $\begin{array}{l}\text { Prel. } \\
\text { E:t. } \\
\text { 1994 }\end{array}$ \\
\hline & \multicolumn{6}{|c|}{ (lo thoundis of metric tons) } \\
\hline A Production & 6,019 & 5,400 & 6218 & 6300 & 5,000 & 5,607 \\
\hline $\begin{array}{l}\text { Crude petroleum } \\
\text { Gas y } \\
\text { Production } \\
\text { Rogatios ? }\end{array}$ & $\begin{array}{r}5,00 \\
97 \\
550\end{array}$ & $\begin{array}{r}4,612 \\
818 \\
274 \\
544\end{array}$ & $\begin{array}{r}5291 \\
921 \\
238 \\
689\end{array}$ & $\begin{array}{r}5,301 \\
1,001 \\
208 \\
763\end{array}$ & $\begin{array}{l}4,7 \% 6 \\
876 \\
165 \\
711\end{array}$ & $\begin{array}{r}4,574 \\
1,083 \\
153 \\
800\end{array}$ \\
\hline B. Consumption & 4,150 & 4,306 & 427 & 4,776 & 5,027 & 4809 \\
\hline $\begin{array}{l}\text { Liquefied petroleum ges } \\
\text { Ges } \\
\text { Fuel oil } \\
\text { Lirpiting oil } \\
\text { Gacolion } \\
\text { Jex fuel } \\
\text { Natural ges y }\end{array}$ & $\begin{array}{l}247 \\
992 \\
887 \\
152 \\
258 \\
191 \\
1,023\end{array}$ & $\begin{array}{r}263 \\
1,063 \\
1,147 \\
147 \\
276 \\
234 \\
1,186\end{array}$ & $\begin{array}{r}280 \\
1,116 \\
1,352 \\
149 \\
291 \\
137 \\
952\end{array}$ & $\begin{array}{r}300 \\
1,160 \\
1,266 \\
158 \\
209 \\
193 \\
1,391\end{array}$ & $\begin{array}{r}312 \\
1,232 \\
1,324 \\
168 \\
309 \\
205 \\
1,47\end{array}$ & $\begin{array}{r}304 \\
1,273 \\
1,126 \\
162 \\
321 \\
248 \\
1,135\end{array}$ \\
\hline \multirow[t]{2}{*}{ Surpines $(A-B)$} & 1,869 & 1,124 & 1,941 & 1,506 & $\operatorname{ses}$ & 718 \\
\hline & \multicolumn{6}{|c|}{ 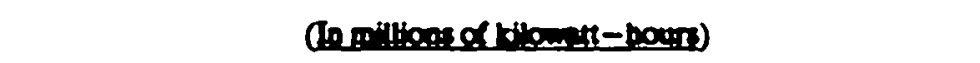 } \\
\hline Ebectricity production $3 /$ & 4,485 & 4,863 & 5,056 & 5,479 & 5,500 & 6,300 \\
\hline
\end{tabular}

Sources: Miniury of National Economy, and Direction geocenle des Minea

VI In thovends of toes of oil equivilent.

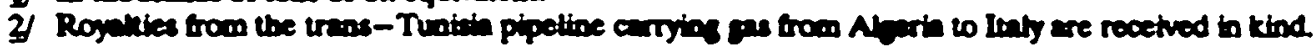

3/ Production by the ante compeny STEG (exctuding production by private plants). 
Table 9. Tunisia: Indicators of Tourism Activity, 1989-94

\begin{tabular}{|c|c|c|c|c|c|c|}
\hline & 1989 & 1990 & 1991 & 1992 & 1993 & Prel. \\
\hline $\begin{array}{l}\text { Annual fixed investment in tourism } \\
\text { (In millions of Tunisian dinars) }\end{array}$ & 109 & 121 & 143 & 250 & 327 & 331 \\
\hline $\begin{array}{l}\text { Accommodation available } \\
\text { (in thousands of beds) }\end{array}$ & 110 & 117 & 123 & 136 & 144 & 158 \\
\hline $\begin{array}{l}\text { Number of foreign visitors } \\
\text { (in thousends) }\end{array}$ & 3,222 & 3,204 & 3,224 & 3,540 & 3,656 & 3,800 \\
\hline $\begin{array}{l}\text { Visitor bed-nights } \\
\text { (in thousands) } \\
\text { Of which: foreigners }\end{array}$ & $\begin{array}{l}20,126 \\
19,011\end{array}$ & $\begin{array}{l}20,019 \\
18,841\end{array}$ & $\begin{array}{l}13,793 \\
12,443\end{array}$ & $\begin{array}{l}21,718 \\
20,206\end{array}$ & $\begin{array}{l}23,693 \\
22,119\end{array}$ & $\begin{array}{l}26,000 \\
24,180\end{array}$ \\
\hline $\begin{array}{l}\text { Average length of stay } \\
\text { (in days) }\end{array}$ & 6 & 6 & 4 & 6 & 6 & 6 \\
\hline $\begin{array}{l}\text { Average occupancy rate } \\
\text { (in percent) }\end{array}$ & 57.6 & 54.0 & 37.6 & 52.0 & 51.6 & 51.0 \\
\hline $\begin{array}{l}\text { Tourism receipts } \\
\text { (in millions of Tunisian dinars) }\end{array}$ & 881 & 828 & 630 & 933 & 1,114 & 1,330 \\
\hline $\begin{array}{l}\text { Daily expenditure per tourist } \\
\text { (in Tunisian dinars) }\end{array}$ & 46 & 44 & 51 & 46 & 50 & 55 \\
\hline
\end{tabular}

Sources: National Tourtst Board; and Central Bank of Tunisia. 
Table 10. Tonida: Compuner Price Index, 1509-94 $y$

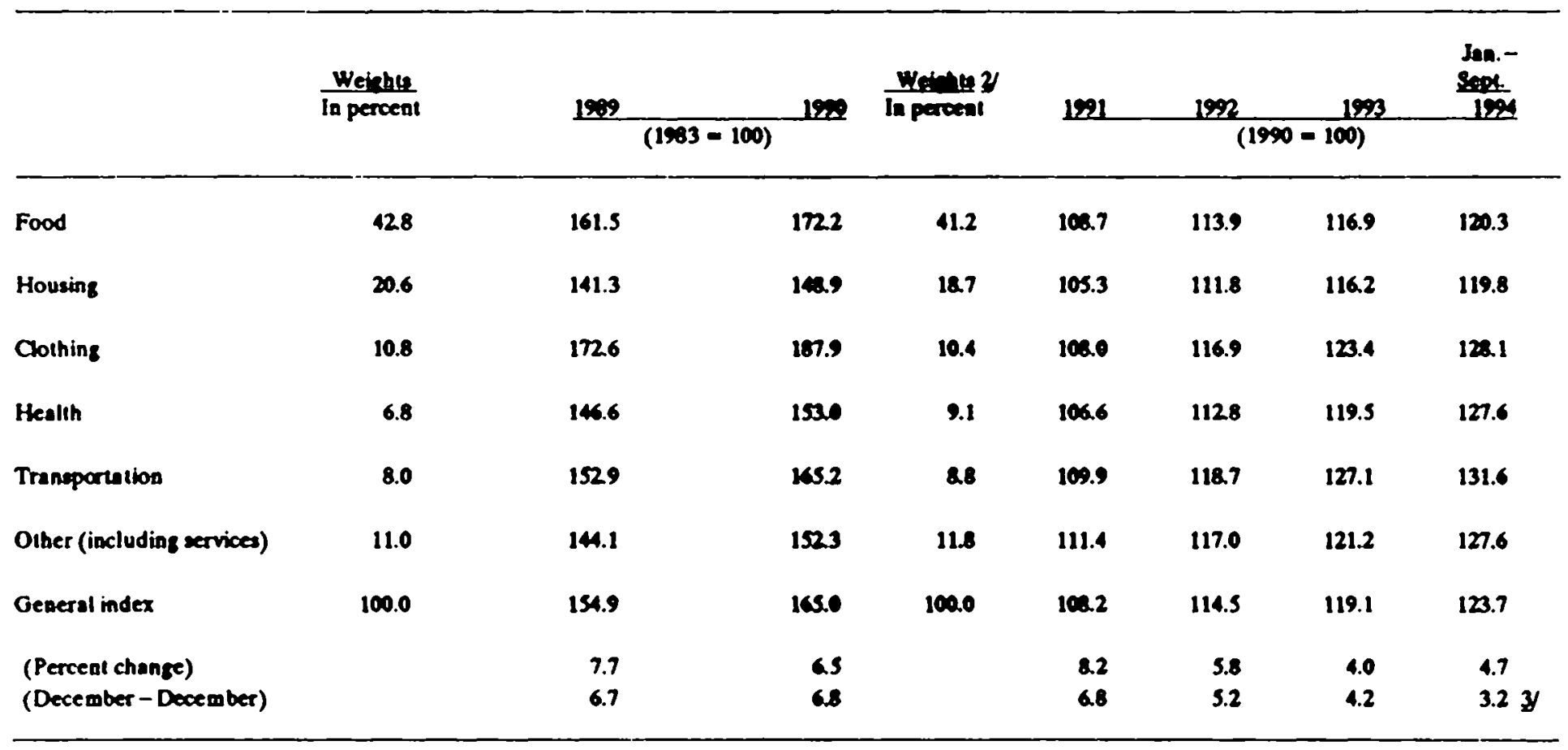

Source: Tunistan autboritke.

1 Pertod averagea.

21 New consuber price index based on recent expeadkure swey.

3. December-September. 
Table 11. Tunisia: Consumer Price Index by Price Regime, 1989-93

\begin{tabular}{|c|c|c|c|c|c|}
\hline Price regime & 1989 & 1990 & 1991 & 1992 & 1993 \\
\hline & \multicolumn{5}{|c|}{ (Chanes Decomber - December) } \\
\hline Fibed prices $y$ & 7.3 & 7.0 & 7.1 & $\cdots$ & $\cdots$ \\
\hline Homologation 2 & 14.8 & 7.8 & 5.3 & 5.8 & 6.4 \\
\hline Autobomologation 3 & 7.2 & 3.6 & 4.5 & 4.8 & 3.3 \\
\hline Controlled freedom $1 / 4$ & 5.7 & 7.7 & 9.7 & $\ldots$ & $\ldots$ \\
\hline Free prices & 6.1 & 72 & 7.4 & 4.8 & 3.0 \\
\hline Total & 6.7 & 6.8 & 7.1 & 5.0 & 4.0 \\
\hline
\end{tabular}

Sounce: Tuniried authoritica.

y Regimes of fored prices and controlled freed on were abolibbed at the beginoing of 1992 .

2 Under bomobontion, prices are set by the Government beed on informetion aubmitted by enterpriver. Through 1991, prices were determined on an infividul enterprive beait; since 1992 , they can be set for all enterpriea in a given sector.

3 Prices are set by enterprives woording to predetermined formula set by the Government.

4 Prices are set by enterprives bened on their ours cost calculations, nubject to Government control 
Table 12. Tunisia: Producer Price Index, 1989-94

\section{(Annual merares; $1983=100$ )}

\begin{tabular}{|c|c|c|c|c|c|c|c|}
\hline & $\frac{\text { Weights }}{\text { In percent }}$ & 1989 & 1990 & 1991 & 1992 & 1993 & $\begin{array}{l}\text { Jan- } \\
\frac{\text { Auk }}{1994}\end{array}$ \\
\hline Manufacturing & S1.1 & 158.8 & 168.3 & 175.3 & 181.4 & 187.7 & 190.9 \\
\hline $\begin{array}{l}\text { Food processing } \\
\text { Construction materials }\end{array}$ & 10.7 & 146.1 & 159.9 & 170.0 & 181.3 & 194.4 & 199.9 \\
\hline $\begin{array}{l}\text { and glass } \\
\text { Mechanical and electrical }\end{array}$ & 7.8 & 137.5 & 144.4 & 158.2 & 166.0 & 169.5 & 174.5 \\
\hline products & 9.6 & 1680 & 180.5 & 185.2 & 185.8 & 188.7 & 189.9 \\
\hline $\begin{array}{l}\text { Chemicals and rubber } \\
\text { Texiles, clothing, and }\end{array}$ & 3.8 & 1624 & 173.6 & 177.1 & 185.1 & 197.8 & 203.9 \\
\hline leather & 12.3 & 164.2 & 169.5 & 174.1 & 180.8 & 185.3 & 187.4 \\
\hline Other manufacturing & 6.9 & 178.1 & 186.3 & 190.5 & 191.8 & 195.2 & 195.6 \\
\hline Mining & 4.4 & 140.0 & 1328 & 140.5 & 128.3 & 127.3 & 125.6 \\
\hline Hydrocarbons & 37.2 & 189.0 & 189.0 & 189.5 & 189.8 & 190.2 & 190.3 \\
\hline Electricity and water & 7.3 & 125.8 & 128.3 & 134.1 & 136.3 & 140.2 & 144.9 \\
\hline General index & 100.0 & 166.8 & 171.5 & 176.0 & 178.9 & 1824 & 184.4 \\
\hline Percent change & & 5.7 & 2.8 & 2.6 & 1.6 & 2.0 & 1.31 \\
\hline
\end{tabular}

Source: Central Bank of Tunisia.

y Over corresponding period of 1993. 
Table 13. Tunisia: Wage and Employment Indicators, 1989-93

\begin{tabular}{|c|c|c|c|c|c|}
\hline & 1989 & 1990 & 1991 & 1992 & 1993 \\
\hline Total wage bill (in millions of Tunisian dinars) & 3,075 & 3,330 & 3,736 & 4,139 & .. \\
\hline Percent change & 11.6 & 8.3 & 12.2 & 10.8 & .. \\
\hline $\begin{array}{l}\text { Number of wage carners } \\
\text { (in thousands) }\end{array}$ & 1,237 & 1,268 & 1,294 & 1,330 & .. \\
\hline $\begin{array}{r}\text { Average annual wage } \\
\text { (in Tunbian dinars) }\end{array}$ & 2,486 & 2,626 & 2,887 & 3,112 & .. \\
\hline Percent change & 8.6 & 5.6 & 9.9 & 7.8 & .. \\
\hline $\begin{array}{l}\text { Real average annual wage } \\
\text { (percent change) } 1 /\end{array}$ & 0.9 & -0.9 & 2.3 & 1.8 & .. \\
\hline SMAO (in Tunisian dinars per day) 2 & 3.213 & 3.661 & 3.761 & 4.261 & 4.361 \\
\hline SMIG (in Tunisian dinars per hour) $\mathfrak{Z}$ & 0.549 & 0.577 & 0.625 & 0.649 & 0.697 \\
\hline $\begin{array}{l}\text { Consumer price index } \\
\text { (percent change) }\end{array}$ & 7.7 & 6.5 & 8.2 & 5.8 & 4.0 \\
\hline
\end{tabular}

Source: Ministry of Planning and Regional Development.

1) Deflated by the average annual increase in the consumer price index.

2) Minimum wage in the agricultural sector.

3) Minimum wage in the nonagricultural sector for full-time emptoyees. 
Table 14. Tunitia: Groes Fibed Irveatmeat by Economic Sector and

Finacing, 1909-94

(In millions o Tumition dimer)

\begin{tabular}{|c|c|c|c|c|c|c|}
\hline & 1980 & 1990 & 1991 & 1992 & 1993 & $\frac{\mathrm{Bg}}{1994}$ \\
\hline Agricullure and fething & 326 & 389 & 418 & 454 & 49 & 584 \\
\hline Induetry & 620 & 749 & 817 & 1,141 & 1,502 & 1601 \\
\hline Mining & 30 & 32 & 33 & 38 & 6 & 43 \\
\hline Hydrocartoons & 119 & 170 & 163 & 489 & 751 & 705 \\
\hline Electricity and wher & 87 & 116 & 122 & 130 & 231 & 325 \\
\hline Manufacturing & 384 & $\$ 11$ & 49 & 483 & 491 & 527 \\
\hline $\begin{array}{l}\text { Food procesting } \\
\text { Coorinction meterials }\end{array}$ & 57 & 73 & 114 & 79 & 81 & 90 \\
\hline $\begin{array}{l}\text { and glam } \\
\text { Mechnical end electrical }\end{array}$ & 54 & 59 & 78 & 80 & 76 & 85 \\
\hline $\begin{array}{l}\text { goods } \\
\text { Coeonical and rubber }\end{array}$ & $\boldsymbol{n}$ & 80 & 89 & 83 & 83 & 84 \\
\hline $\begin{array}{l}\text { products } \\
\text { Textiles and leather }\end{array}$ & 29 & 32 & 36 & 40 & 55 & 63 \\
\hline products & 135 & 140 & 137 & 135 & 132 & 135 \\
\hline $\begin{array}{l}\text { Woodwort and otber } \\
\text { Conetruction ind public }\end{array}$ & 39 & 46 & 46 & 66 & 63 & 70 \\
\hline infirestructure & 663 & $\pi 4$ & 845 & 923 & 1,027 & 1,1228 \\
\hline $\begin{array}{l}\text { Houring } \\
\text { Contruction and public }\end{array}$ & 398 & 454 & 500 & 555 & 608 & 60 \\
\hline $\begin{array}{l}\text { wartos } \\
\text { Public infratructure }\end{array}$ & $\begin{array}{r}36 \\
230\end{array}$ & $\begin{array}{r}50 \\
270\end{array}$ & $\begin{array}{r}59 \\
286\end{array}$ & $\begin{array}{r}33 \\
335\end{array}$ & 39 & $\begin{array}{r}48 \\
40\end{array}$ \\
\hline $\begin{array}{l}\text { Services } \\
\text { Trapeport and coon- }\end{array}$ & 548 & 223 & 833 & 1,117 & 1,112 & 1,345 \\
\hline munications & 292 & 410 & 469 & 611 & 575 & 767 \\
\hline Logding, food and beverages & 100 & 118 & 137 & 254 & 290 & 305 \\
\hline Commerce and ouber & 156 & 195 & 227 & 223 & 247 & 274 \\
\hline Tocal & 2,157 & 2,635 & 2,921 & 3,035 & 4,168 & 4,658 \\
\hline $\begin{array}{l}\text { Central governmeot } \\
\text { Private sector } y\end{array}$ & $\begin{array}{r}403 \\
1,754\end{array}$ & 2,137 & $\begin{array}{r}697 \\
2204\end{array}$ & $\begin{array}{r}560 \\
3,075\end{array}$ & $\begin{array}{r}653 \\
3,515\end{array}$ & $\begin{array}{r}744 \\
3,914\end{array}$ \\
\hline $\begin{array}{l}\text { Finaccing requirements } 2 / \\
\text { Jrow national guvings } \\
\text { (in percent o (GDP) }\end{array}$ & $\begin{array}{r}2,258 \\
1,914 \\
20.1\end{array}$ & $\begin{array}{r}2,877 \\
2,308 \\
21.4\end{array}$ & $\begin{array}{r}3,144 \\
2579 \\
21.5\end{array}$ & $\begin{array}{r}4,131 \\
3,214 \\
23.4\end{array}$ & $\begin{array}{r}4,308 \\
3,145 \\
21.4\end{array}$ & $\begin{array}{r}4,740 \\
3,600 \\
224\end{array}$ \\
\hline $\begin{array}{l}\text { Foreign resources } \\
\text { (in percent of GDP) }\end{array}$ & $\begin{array}{r}344 \\
3.6\end{array}$ & $\begin{array}{r}569 \\
5.3\end{array}$ & $\begin{array}{r}565 \\
4.7\end{array}$ & $\begin{array}{r}917 \\
6.7\end{array}$ & $\begin{array}{r}1163 \\
7.9\end{array}$ & $\begin{array}{r}1120 \\
6.9\end{array}$ \\
\hline
\end{tabular}

Source: Miniary of Planoing and Regional Development.

$\because$ Includes all economic agents except the Central Government.

2i. includes changes in stoctes. 
Table 15. Tunisia: Consolidated Financial Operations of the Central Government, 1989-94 $1 /$

\begin{tabular}{|c|c|c|c|c|c|c|}
\hline & 1989 & 1990 & 1991 & 1992 & 1993 & $\begin{array}{l}\text { Prel. } \\
\frac{\text { Est. }}{1994}\end{array}$ \\
\hline & \multicolumn{6}{|c|}{ (In millioss of Tunisian dinass) } \\
\hline $\begin{array}{l}\text { Total revenue and grants } \\
\text { Toul revenue } \\
\text { Tax revenue } \\
\text { Taxes on foreiga trade } \\
\text { Other taxes } \\
\text { Noatax revenue } \\
\text { Petroleum sector } \\
\text { Other nontax revenue } \\
\text { Grants }\end{array}$ & $\begin{array}{r}3,071 \\
2,928 \\
2,258 \\
817 \\
1,441 \\
670 \\
394 \\
276 \\
143\end{array}$ & $\begin{array}{r}3,356 \\
3,286 \\
2,551 \\
889 \\
1,663 \\
735 \\
393 \\
342 \\
70\end{array}$ & $\begin{array}{r}3,524 \\
3,491 \\
2,875 \\
955 \\
1,920 \\
617 \\
254 \\
362 \\
33\end{array}$ & $\begin{array}{r}4,085 \\
4,031 \\
3,294 \\
1,112 \\
2,182 \\
737 \\
296 \\
441 \\
54\end{array}$ & $\begin{array}{r}4,488 \\
4,451 \\
3,625 \\
1,162 \\
2,463 \\
826 \\
260 \\
566 \\
37\end{array}$ & $\begin{array}{r}4,923 \\
4,886 \\
4,044 \\
\ldots \\
\ldots 42 \\
250 \\
592 \\
37\end{array}$ \\
\hline $\begin{array}{l}\text { Total expenditure and net lending } \\
\text { Total expenditure } \\
\text { Current expenditure } \\
\text { Wages and salaries } \\
\text { Goods and services } \\
\text { Intereat payments } \\
\text { Foreign debt } \\
\text { Domeatic debt } \\
\text { Subidies and transfers } \\
\text { Of which: coneumer subsidies } \\
\text { Capital expenditure } \\
\text { Direct imventment } \\
\text { Capital tramiers and equity } \\
\text { Net lending }\end{array}$ & $\begin{array}{r}3,483 \\
3,418 \\
2,693 \\
950 \\
208 \\
317 \\
239 \\
78 \\
1,219 \\
346 \\
725 \\
403 \\
322 \\
65\end{array}$ & $\begin{array}{r}3,787 \\
3,743 \\
2,863 \\
1,014 \\
231 \\
341 \\
249 \\
92 \\
1,277 \\
300 \\
880 \\
498 \\
383 \\
44\end{array}$ & $\begin{array}{r}4,179 \\
4,202 \\
3,176 \\
1,158 \\
286 \\
416 \\
258 \\
158 \\
1,316 \\
272 \\
1,026 \\
697 \\
329 \\
-24\end{array}$ & $\begin{array}{r}4,445 \\
4,381 \\
3,490 \\
1,302 \\
289 \\
435 \\
247 \\
188 \\
1,465 \\
255 \\
891 \\
560 \\
331 \\
64\end{array}$ & $\begin{array}{r}4,914 \\
4,849 \\
3,835 \\
1,476 \\
293 \\
511 \\
281 \\
231 \\
1,555 \\
245 \\
1,014 \\
653 \\
361 \\
65\end{array}$ & $\begin{array}{r}5,304 \\
5,332 \\
4,210 \\
1,614 \\
299 \\
520 \\
322 \\
198 \\
1,747 \\
230 \\
1,122 \\
744 \\
378 \\
-28\end{array}$ \\
\hline Overall deficit & -412 & -431 & -655 & -360 & -426 & -382 \\
\hline $\begin{array}{l}\text { Financing } \\
\text { Foreige } \\
\text { Drawing } \\
\text { Of which: direct financing } \\
\text { Amortination } \\
\text { Domestic } \\
\text { Banks } \\
\text { Other } 2\end{array}$ & $\begin{array}{r}412 \\
118 \\
521 \\
210 \\
-403 \\
294 \\
45 \\
249\end{array}$ & $\begin{array}{r}431 \\
75 \\
538 \\
255 \\
-463 \\
356 \\
85 \\
271\end{array}$ & $\begin{array}{r}655 \\
319 \\
799 \\
361 \\
-480 \\
336 \\
45 \\
291\end{array}$ & $\begin{array}{r}360 \\
22 \\
579 \\
230 \\
-557 \\
337 \\
-114 \\
451\end{array}$ & $\begin{array}{r}426 \\
178 \\
695 \\
274 \\
-517 \\
248 \\
-47 \\
295\end{array}$ & $\begin{array}{r}382 \\
144 \\
725 \\
215 \\
-581 \\
238 \\
-14 \\
252\end{array}$ \\
\hline $\begin{array}{l}\text { Memorandum items: } \\
\text { GDP } \\
\text { Overall deficit, excluding grants } \\
\text { Military expenditure }\end{array}$ & $\begin{array}{r}9,531 \\
-555 \\
188\end{array}$ & $\begin{array}{r}10,798 \\
-501 \\
225\end{array}$ & $\begin{array}{r}11,997 \\
-687 \\
257 \\
\\
\text { (In pers }\end{array}$ & $\begin{array}{r}13,754 \\
-414 \\
260 \\
\\
\text { (GDP) }\end{array}$ & $\begin{array}{r}14,688 \\
-463 \\
279\end{array}$ & $\begin{array}{r}16,134 \\
-418 \\
289\end{array}$ \\
\hline $\begin{array}{l}\text { Revenue and grants } \\
\text { Tax revenue } \\
\text { Grants } \\
\text { Expenditure and net lending } \\
\text { Current expenditure } \\
\text { Capital expenditure } \\
\text { Deficit including grants } \\
\text { Deficit excluding grants }\end{array}$ & $\begin{array}{r}32.2 \\
23.7 \\
1.5 \\
36.5 \\
28.3 \\
7.6 \\
-4.3 \\
-5.8\end{array}$ & $\begin{array}{r}31.1 \\
23.6 \\
0.6 \\
35.1 \\
26.5 \\
8.2 \\
-4.0 \\
-4.6\end{array}$ & $\begin{array}{r}29.4 \\
24.0 \\
0.3 \\
34.8 \\
26.5 \\
8.6 \\
-5.5 \\
-5.7\end{array}$ & $\begin{array}{r}29.7 \\
23.9 \\
0.4 \\
32.3 \\
25.4 \\
6.5 \\
-2.6 \\
-3.0\end{array}$ & $\begin{array}{r}30.6 \\
24.7 \\
0.3 \\
33.5 \\
26.1 \\
6.9 \\
-2.9 \\
-32\end{array}$ & $\begin{array}{r}30.5 \\
25.1 \\
0.2 \\
32.9 \\
26.1 \\
7.0 \\
-2.4 \\
-2.6\end{array}$ \\
\hline
\end{tabular}

Sources: Ministry of Finance; and Fund staff estimates.

1/ Includes current and capital budgets, Special Funds, Fonds de Concours, extrabudgetary operations financed abroad, net treasury operations, and social security funds.

2) Including payment float, errors, and omissions. 
Table 16. Tunitia: Coacolstand Reviave sod Orants of the Ceotral Governmeot, 1909-93

(In milligas of Runinindinars)

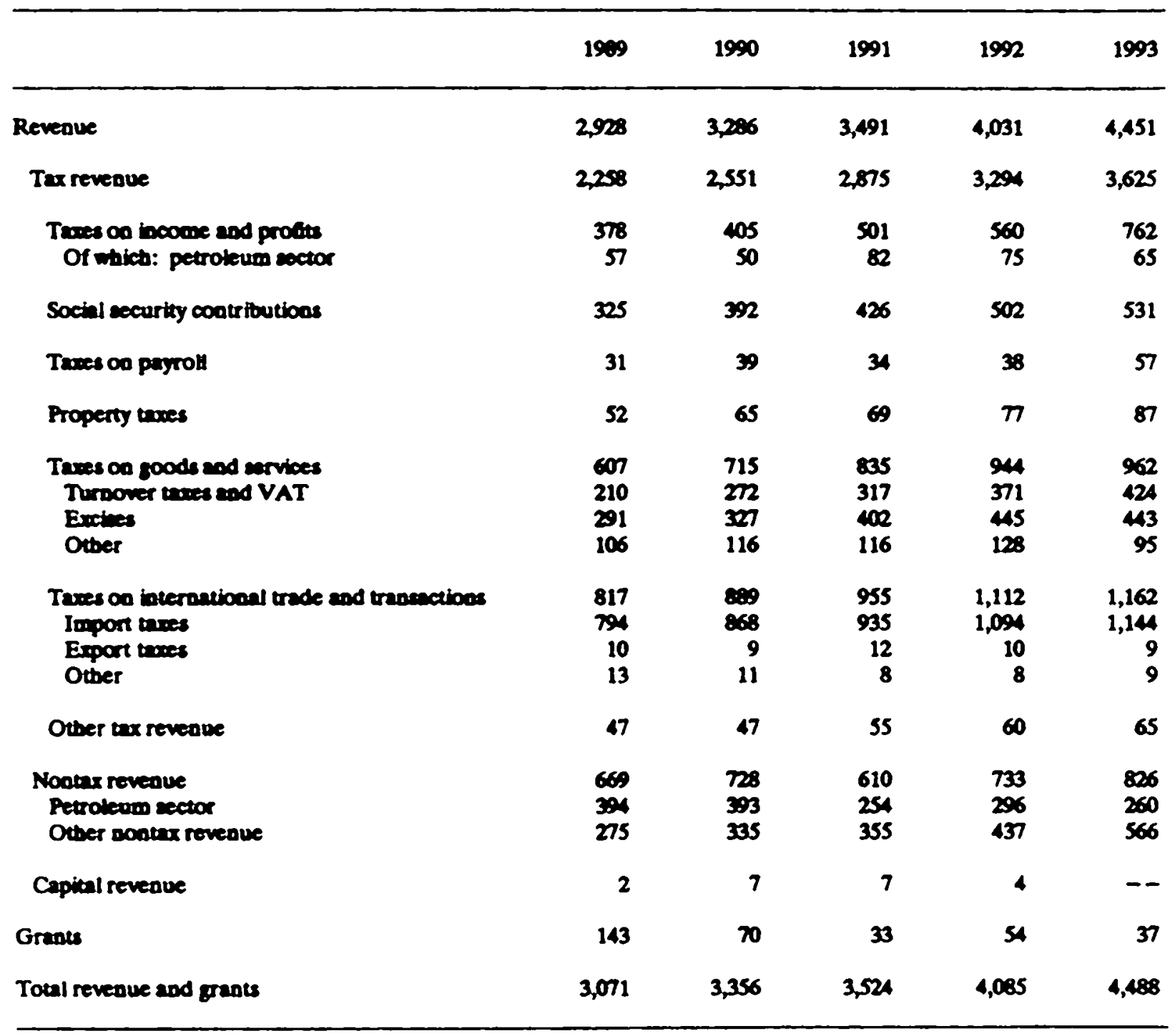

Source: Miniatry of Finance. 
Table 17. Tunisia: Government Petroleum Revenues, 1989-94

(In millions of Tunisian dinars)

\begin{tabular}{|c|c|c|c|c|c|c|}
\hline & 1989 & 1990 & 1991 & 1992 & 1993 & $\begin{array}{l}\text { Prel. } \\
\text { Bst. } \\
1994\end{array}$ \\
\hline $\begin{array}{l}\text { Profits from petroleum exploitation } \\
\text { (Title I, Articles } 51.02 \text { and } 51.03 \text { ) }\end{array}$ & 394 & 393 & 254 & 296 & 260 & 235 \\
\hline $\begin{array}{l}\text { Excises on refined petroleum products } \\
\text { (Title I, Article 21.07) }\end{array}$ & 99 & 107 & 161 & 171 & 178 & 230 \\
\hline Overall total & 588 & 593 & 558 & 597 & 572 & 611 \\
\hline
\end{tabular}

Sources: Ministry of Finance; and Fund staff estimates. 
Table 18. Tuniaia: Economic Cbasification of Comolidated Expenditure of the Central Government, 1969-94 $y$

(In millyone of Tunthe dinn )

\begin{tabular}{|c|c|c|c|c|c|c|}
\hline & 1989 & 1990 & 1991 & 1992 & 1993 & $\begin{array}{l}\text { Prel } \\
\text { Ext. } \\
1994\end{array}$ \\
\hline Current expenditure & 2,693 & 2,863 & 3,176 & 3,490 & 3,835 & 4,210 \\
\hline Wages and salaries & 950 & 1,014 & 1,158 & 1,302 & 1,476 & 1,644 \\
\hline Goods and other services & 208 & 231 & 286 & 289 & 293 & 299 \\
\hline Interest on public debt & 317 & 341 & 416 & 435 & $S 11$ & 520 \\
\hline Forelga debt & 239 & 249 & 258 & 247 & 281 & 322 \\
\hline Domestic debt & 78 & $\boldsymbol{\Omega}$ & 158 & 188 & 231 & 198 \\
\hline $\begin{array}{l}\text { Subsidies and other current transfers } \\
\text { Of which: }\end{array}$ & 1,219 & 1,277 & 1,316 & 1,465 & 1,555 & 1,747 \\
\hline Consumer substdies & 346 & 300 & 272 & 255 & 245 & 230 \\
\hline Capital expenditure & T2S & 880 & 1,026 & 891 & 1,014 & 1,122 \\
\hline Direct investment & 403 & 498 & 697 & 560 & 653 & 744 \\
\hline Capital transfers and equity & 322 & 383 & 329 & 331 & 361 & 378 \\
\hline Total expenditure & 3,418 & 3,743 & 4,202 & 4,381 & 4,849 & 5,332 \\
\hline
\end{tabular}

Sources: Ministry of Finance; and Fund staff estimates.

1/ Includes all expenditure in the treasury acoounts, extrabudgetary operations financed by external assistance, and expenditure of the social security schemes. 
Table 19: Tunisia: Opermions of the Subudy Fund, 1969-94

\section{(Inmilliom of Tunielan diners)}

\begin{tabular}{|c|c|c|c|c|c|c|}
\hline & 1989 & 1990 & 1991 & 1992 & 1993 & $\begin{array}{l}\text { Prel. } \\
\text { Pet. } \\
\frac{1994}{}\end{array}$ \\
\hline Subsidies and other expenditures 1 & 334 & 319 & 285 & 274 & 260 & 258 \\
\hline Fertilizer & 21 & 17 & 12 & 5 & 1 & -- \\
\hline Sugar & 35 & 35 & 18 & 14 & 15 & 16 \\
\hline Cereals & 200 & 180 & 165 & 165 & 165 & 159 \\
\hline Vegetable oil & 25 & 35 & 43 & 40 & 35 & 40 \\
\hline Milk & 35 & 8 & 11 & 24 & 22 & 19 \\
\hline Reimbursement of treaury loans & 5 & 5 & 5 & $\mathbf{s}$ & $\ldots$ & - \\
\hline Miscellaneous expenditures & 13 & 39 & 31 & 21 & 22 & 25 \\
\hline Tocal resources & 334 & 319 & 287 & 276 & 265 & 258 \\
\hline Earmarked taxes $y$ & 18 & -- & -- & -- & -- & - \\
\hline $\begin{array}{l}\text { Curremt budiget tranafers } \\
\text { Borrowing from Treasury and }\end{array}$ & 231 & 300 & 272 & 255 & 245 & 230 \\
\hline transfers from tressury funds & 22 & 1 & -- & $-\infty$ & -- & -- \\
\hline Grants & 62 & -- & -- & -- & - & - \\
\hline Revaluation profits on atocks & 1 & 1 & 1 & 1 & 1 & 1 \\
\hline Other & 1 & 17 & 15 & 20 & 19 & 27 \\
\hline Overall surplus or deficil (-) & -- & -- & 2 & 2 & 5 & -- \\
\hline
\end{tabular}

Source: Minisry of Finance.

1) On an sccrual basis, Including the expenditures directly financed through treasury advances.

2 Includes net position of Subsidy Fund with importers, producers, and incermediaries involved is transections of subsidized commodities. 
Table 20. Tunisia: Monetary Survey, 1989-94

(In millions of Tunisian dinars; end of period) .

\begin{tabular}{|c|c|c|c|c|c|c|}
\hline & 1989 & 1990 & 1991 & 1992 & 1993 & $\frac{\text { June }}{1994}$ \\
\hline $\begin{array}{l}\text { Foreign ascets (net) } \\
\text { Foreign aacets } \\
\text { Foreign liabilities }\end{array}$ & $\begin{array}{r}668 \\
1,254 \\
-586\end{array}$ & $\begin{array}{r}612 \\
1,138 \\
-526\end{array}$ & $\begin{array}{r}526 \\
1,193 \\
-667\end{array}$ & $\begin{array}{r}547 \\
1,381 \\
-834\end{array}$ & $\begin{array}{r}563 \\
1,500 \\
-937\end{array}$ & $\begin{array}{r}400 \\
1,533 \\
-1,133\end{array}$ \\
\hline $\begin{array}{l}\text { Domeatic credit } \\
\text { Credit to the Government (net) } \\
\text { Central Bank } \\
\text { Deposit money banks } \\
\text { Counterpart of CCP deposits }\end{array}$ & $\begin{array}{r}5,563 \\
722 \\
-85 \\
715 \\
92\end{array}$ & $\begin{array}{r}6,143 \\
808 \\
-55 \\
762 \\
100\end{array}$ & $\begin{array}{r}6,741 \\
853 \\
-68 \\
793 \\
128\end{array}$ & $\begin{array}{r}7,478 \\
687 \\
47 \\
537 \\
104\end{array}$ & $\begin{array}{r}8,004 \\
640 \\
-6 \\
536 \\
110\end{array}$ & $\begin{array}{r}8,298 \\
507 \\
-73 \\
471 \\
110\end{array}$ \\
\hline $\begin{array}{l}\text { Credit to the economy } \\
\text { Central Bank } \\
\text { Deposit money banks }\end{array}$ & $\begin{array}{r}4,841 \\
31 \\
4,810\end{array}$ & $\begin{array}{r}5,335 \\
34 \\
5,301\end{array}$ & $\begin{array}{r}5,888 \\
41 \\
5,847\end{array}$ & $\begin{array}{r}6,791 \\
39 \\
6,751\end{array}$ & $\begin{array}{r}7,364 \\
26 \\
7,338\end{array}$ & $\begin{array}{r}7,791 \\
24 \\
7,767\end{array}$ \\
\hline $\begin{array}{l}\text { Money plus quasi-money (M2) } \\
\text { Money } \\
\text { Currency } \\
\text { Demand deposits } \\
\text { Finnncial inctitutions } \\
\text { Nonfinancial enterprises }\end{array}$ & $\begin{array}{r}4,910 \\
2,527 \\
875 \\
1,652 \\
37\end{array}$ & $\begin{array}{r}5,200 \\
2,649 \\
1,005 \\
1,644 \\
11\end{array}$ & $\begin{array}{r}5,507 \\
2,640 \\
1,104 \\
1,536 \\
12\end{array}$ & $\begin{array}{r}5,903 \\
2,781 \\
1,156 \\
1,625 \\
14\end{array}$ & $\begin{array}{r}6,294 \\
2,916 \\
1,179 \\
1,736 \\
10\end{array}$ & $\begin{array}{r}6,216 \\
2,954 \\
1,238 \\
1,717 \\
13\end{array}$ \\
\hline $\begin{array}{l}\text { and households } \\
\text { Qunsi-money } \\
\text { Finencial inctitutions } \\
\text { Nonfinancial enterprises } \\
\text { and houreholds }\end{array}$ & $\begin{array}{r}1,615 \\
2,383 \\
73\end{array}$ & $\begin{array}{r}1,633 \\
2,571 \\
108\end{array}$ & $\begin{array}{r}1,524 \\
2867 \\
100\end{array}$ & $\begin{array}{r}1,611 \\
3,122 \\
97\end{array}$ & $\begin{array}{r}1,727 \\
3,378 \\
145\end{array}$ & $\begin{array}{r}1,704 \\
3,262 \\
93\end{array}$ \\
\hline Long-term deposits & 254 & 321 & 351 & 388 & 429 & 441 \\
\hline $\begin{array}{l}\text { Other items (net) } \\
\text { Special resources } \\
\text { Capital accounts } \\
\text { Other }\end{array}$ & $\begin{array}{r}1,067 \\
664 \\
574 \\
-171\end{array}$ & $\begin{array}{r}1214 \\
734 \\
641 \\
-162\end{array}$ & $\begin{array}{r}1,410 \\
806 \\
759 \\
-155\end{array}$ & $\begin{array}{r}1,734 \\
849 \\
942 \\
-57\end{array}$ & $\begin{array}{r}1,844 \\
903 \\
1,113 \\
-172\end{array}$ & $\begin{array}{r}2,041 \\
956 \\
1,256 \\
-171\end{array}$ \\
\hline
\end{tabular}

Sources: Central Bank of Tunisia, Statiatiques Financieres, various iseues; and data communicated by the Central Bank of Tunisia. 
Table 21. Tunisia: Asects and Liabilities of the Central Bank, 1989-94

(In million of Tuninian dinare; end of period)

\begin{tabular}{|c|c|c|c|c|c|c|}
\hline & 1989 & 1990 & 1991 & 1992 & 1993. & $\frac{\text { June }}{1994}$ \\
\hline $\begin{array}{l}\text { Foreign assets } \\
\text { Claims on Government } \\
\text { Claims on development bants } \\
\text { Claims on deposit money banlos }\end{array}$ & $\begin{array}{r}844 \\
27 \\
946\end{array}$ & $\begin{array}{r}633 \\
64 \\
51 \\
855\end{array}$ & $\begin{array}{r}679 \\
95 \\
89 \\
1,037\end{array}$ & $\begin{array}{r}877 \\
117 \\
84 \\
986\end{array}$ & $\begin{array}{r}944 \\
122 \\
95 \\
1,068\end{array}$ & $\begin{array}{r}981 \\
62 \\
61 \\
1,265\end{array}$ \\
\hline Assets = Liabilities & 1,871 & 1,603 & 1,900 & 2,064 & 2,229 & 2,369 \\
\hline $\begin{array}{l}\text { Reserve moncy } \\
\text { Currency outside bants } \\
\text { Currency with bants } \\
\text { Banks' deposits } \\
\text { Claims of development banks and } \\
\text { other financial institutions } \\
\text { Deposits of nonfinancial entities }\end{array}$ & $\begin{array}{r}1,361 \\
875 \\
28 \\
455\end{array}$ & $\begin{array}{r}1,145 \\
1,005 \\
44 \\
89\end{array}$ & $\begin{array}{r}1,264 \\
1,104 \\
43 \\
89\end{array}$ & $\begin{array}{r}1,355 \\
1,156 \\
40 \\
134\end{array}$ & $\begin{array}{r}1,421 \\
1,179 \\
51 \\
157\end{array}$ & $\begin{array}{r}1,576 \\
1,238 \\
50 \\
254\end{array}$ \\
\hline $\begin{array}{l}\text { Foreign liabilities } \\
\text { Government deposits and } \\
\text { currency holdings } \\
\text { Counterpart funds } \\
\text { Allocation of SDRs } \\
\text { Capital sccounts } \\
\text { Other items (net) }\end{array}$ & $\begin{array}{r}259 \\
139 \\
94 \\
20 \\
44 \\
-46\end{array}$ & $\begin{array}{r}119 \\
66 \\
20 \\
49 \\
57\end{array}$ & $\begin{array}{r}164 \\
70 \\
43 \\
49 \\
69\end{array}$ & $\begin{array}{r}71 \\
64 \\
45 \\
54 \\
174\end{array}$ & $\begin{array}{r}128 \\
79 \\
49 \\
59 \\
171\end{array}$ & $\begin{array}{r}135 \\
81 \\
49 \\
69 \\
103\end{array}$ \\
\hline
\end{tabular}

Sources: Central Bank of Tunisia, Statistiques Financieres, various insues. 


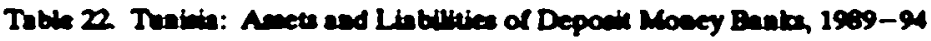

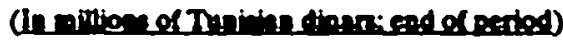

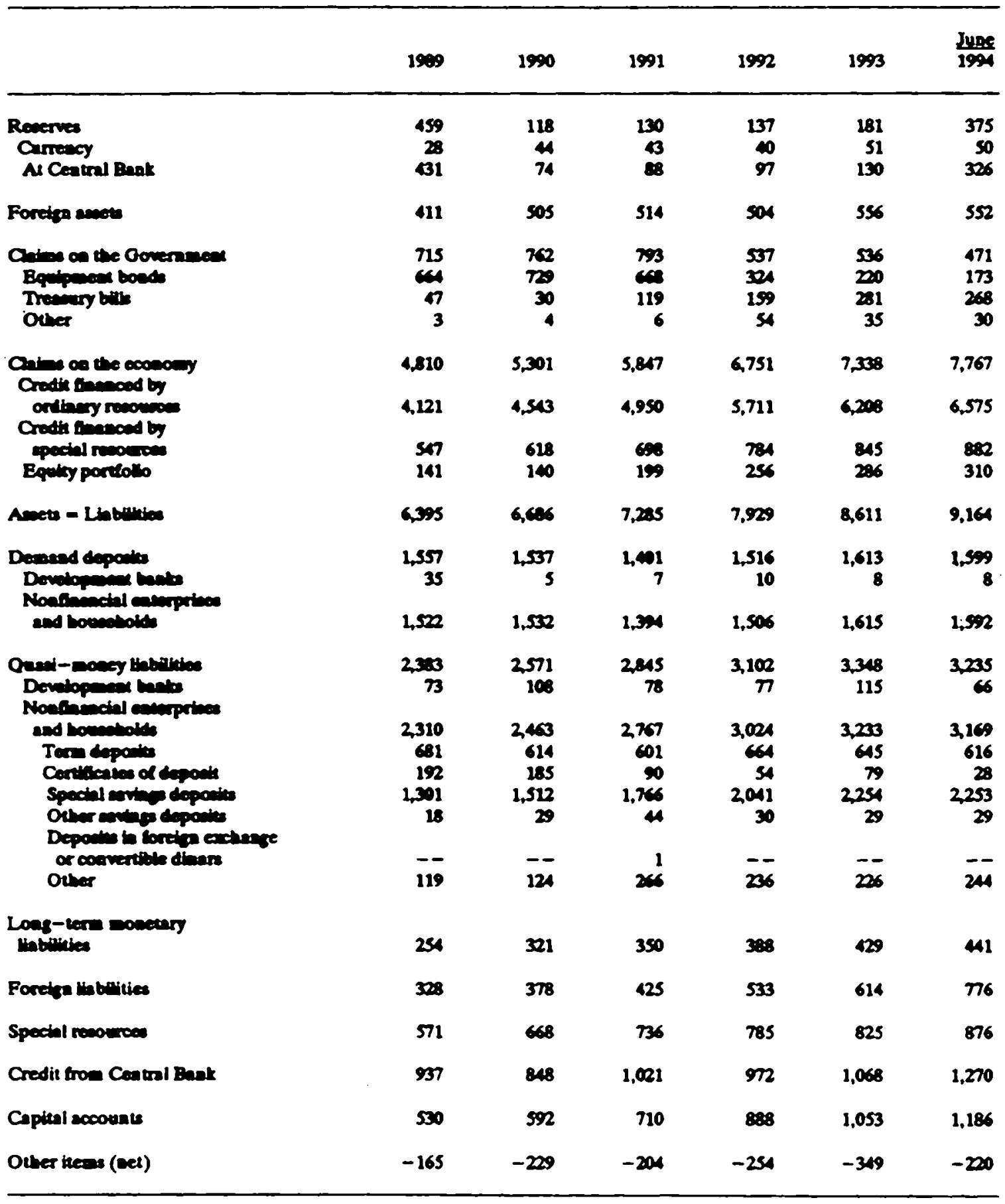

Sourca: Ceatral Bask of Toniwh, Statininua Francites various ineses. 
Table 23. Tunidia: Sefected Intereat Rates, 1900-94

\section{(In percent; end of period)}

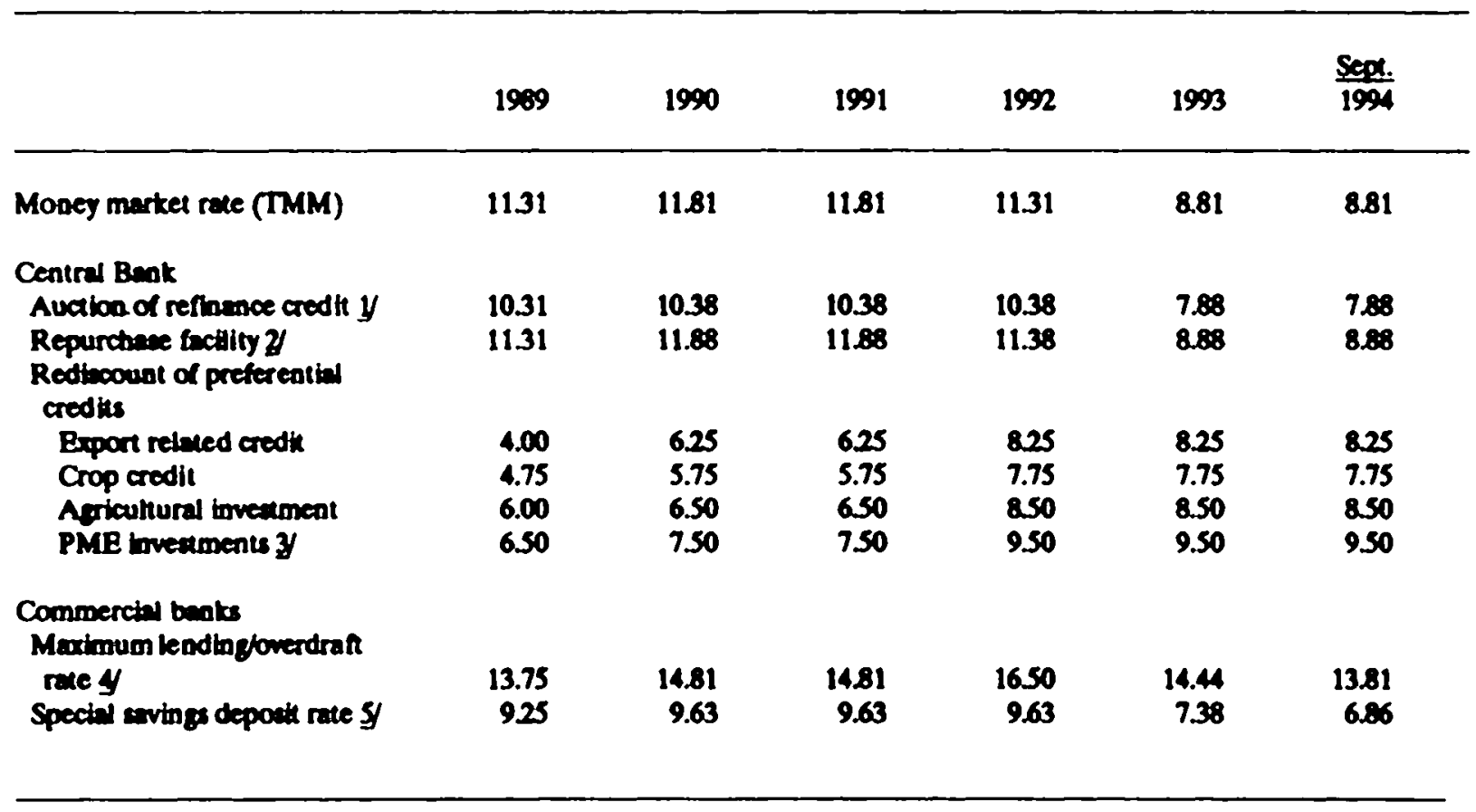

Source: Central Bank of Tunitia.

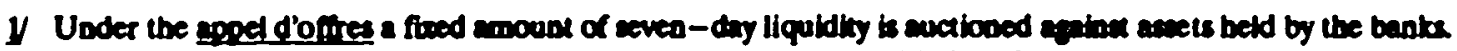

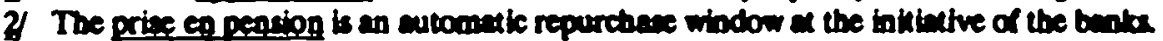

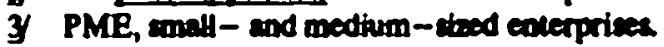

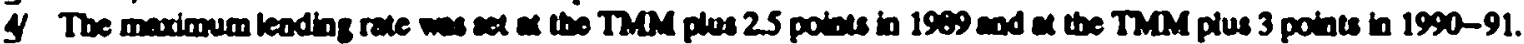

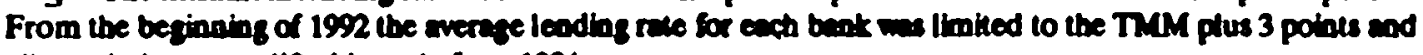
all reatrictions were lined in earty fune 1994.

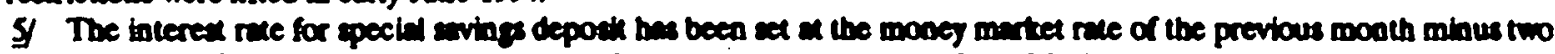
potats stace 1987. Rates on depoits with terms of a leat three months are free. Mroimum rate on deposits of thorter maturities was set at 2 percent in July 1990. 


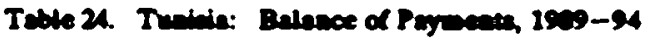

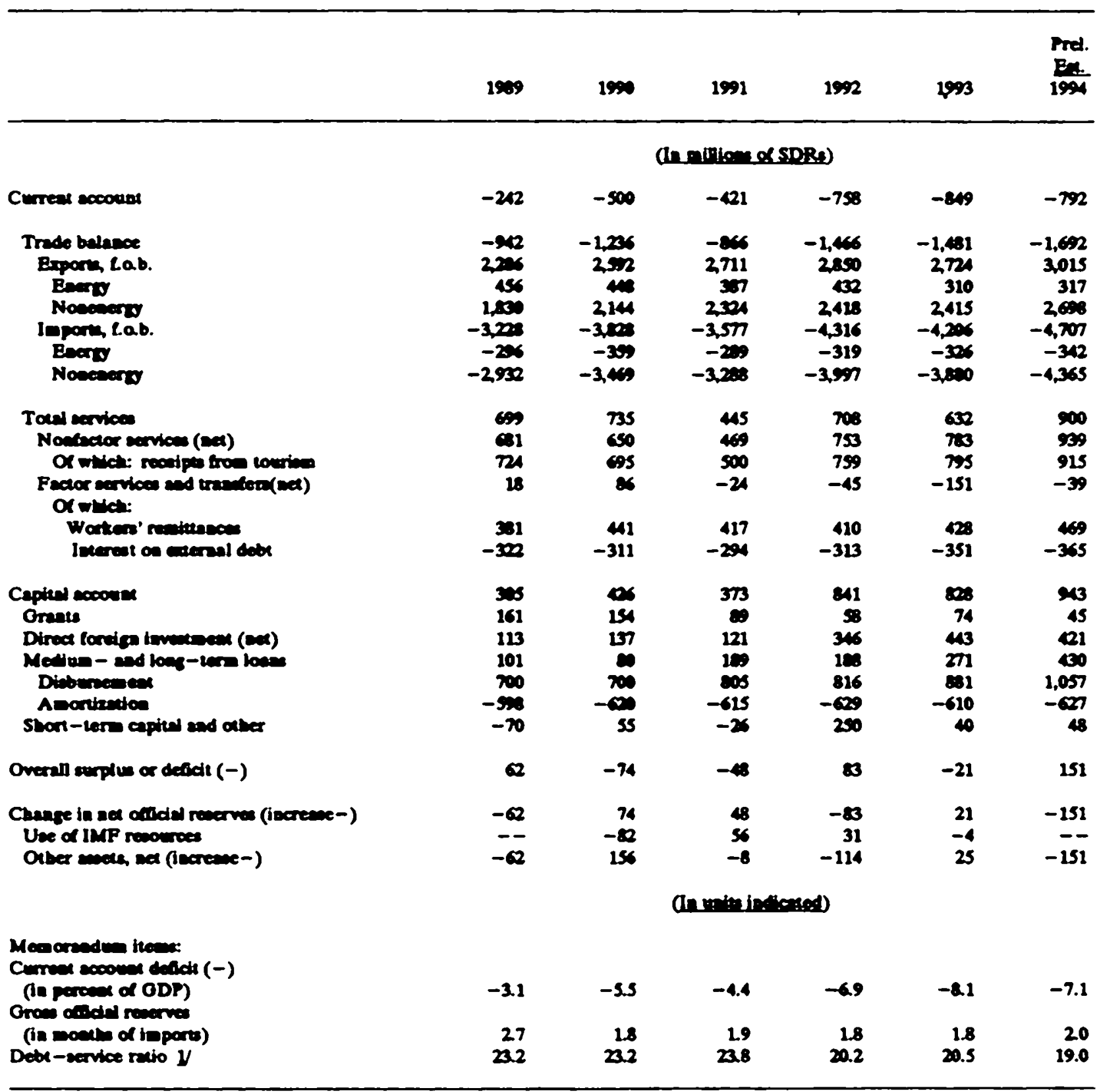

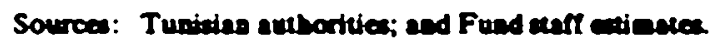

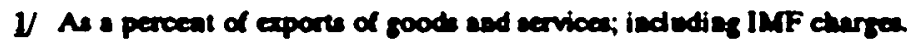


Table 25. Tuniria: Foreign Trade Indicators, 1990-94

(Changes in percent)

\begin{tabular}{|c|c|c|c|c|c|}
\hline & 1990 & 1991 & 1992 & 1993 & 1994 \\
\hline \multicolumn{6}{|l|}{ Exports $y$} \\
\hline $\begin{array}{l}\text { Vahe (in Tunivion dionrs) } \\
\text { Volume } \\
\text { Price }\end{array}$ & $\begin{array}{r}11.0 \\
4.5 \\
6.3\end{array}$ & $\begin{array}{r}11.1 \\
4.4 \\
6.4\end{array}$ & $\begin{array}{l}3.5 \\
3.1 \\
0.3\end{array}$ & $\begin{array}{l}7.6 \\
0.3 \\
7.3\end{array}$ & $\begin{array}{r}14.6 \\
112 \\
3.0\end{array}$ \\
\hline $\begin{array}{l}\text { Vahe (in SDRs) } \\
\text { Price }\end{array}$ & $\begin{array}{r}13.4 \\
8.5\end{array}$ & $\begin{array}{l}4.6 \\
0.2\end{array}$ & $\begin{array}{l}5.1 \\
1.9\end{array}$ & $\begin{array}{l}-4.4 \\
-4.7\end{array}$ & $\begin{array}{r}10.7 \\
-0.5\end{array}$ \\
\hline \multicolumn{6}{|l|}{ Import,cif $y$} \\
\hline $\begin{array}{l}\text { Vahe (in Tuninien dimars) } \\
\text { Vohume } \\
\text { Price }\end{array}$ & $\begin{array}{r}16.9 \\
9.6 \\
6.7\end{array}$ & $\begin{array}{r}-1.3 \\
-7.9 \\
7.1\end{array}$ & $\begin{array}{r}18.8 \\
15.9 \\
2.5\end{array}$ & $\begin{array}{l}9.6 \\
2.6 \\
6.9\end{array}$ & $\begin{array}{r}15.6 \\
11.4 \\
3.8\end{array}$ \\
\hline $\begin{array}{l}\text { Value (in SDR) } \\
\text { Price }\end{array}$ & $\begin{array}{r}19.4 \\
8.9\end{array}$ & $\begin{array}{r}-70 \\
09\end{array}$ & $\begin{array}{r}20.6 \\
4.1\end{array}$ & $\begin{array}{l}-2.6 \\
-5.0\end{array}$ & $\begin{array}{r}11.7 \\
0.3\end{array}$ \\
\hline Terms of trade & -0.4 & -0.7 & -2.1 & 0.4 & -0.8 \\
\hline
\end{tabular}

Source: Ministry of Phaniog and Regional Development.

y Curreat price data are based on custom trade atatiotica. 


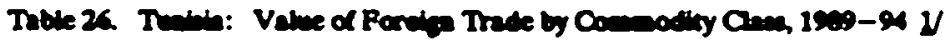

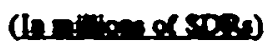

\begin{tabular}{|c|c|c|c|c|c|c|}
\hline & 1900 & 1900 & 1991 & 1992 & 1993 & $\begin{array}{l}\text { Prel } \\
\text { Bet } \\
199\end{array}$ \\
\hline Beparte, tab. & 2,286 & 259 & 2,711 & 2850 & 2,724 & 3015 \\
\hline Benos product: & 456 & 48 & 387 & 430 & 310 & 317 \\
\hline Nowasery & 1,200 & 2,144 & 2.304 & 2,418 & 2,415 & 20 \\
\hline Nopitame and dantion & 47 & 391 & 308 & 395 & 336 & 372 \\
\hline 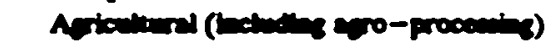 & 202 & 203 & 412 & 236 & 313 & 302 \\
\hline Terionad lowe & 79 & $\mathbf{3 3}$ & 1,060 & 1,231 & 1,268 & 1,450 \\
\hline 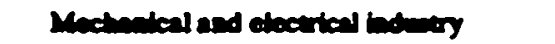 & 191 & 29 & 300 & 341 & 302 & 390 \\
\hline Oder goode & 241 & 179 & 154 & 156 & 136 & 115 \\
\hline Inportinall & $\mathbf{3 , 4 1 1}$ & 400 & 3,706 & 4,597 & 4,450 & 4,970 \\
\hline Beng produce & 206 & 35 & 2 & 319 & 305 & 30 \\
\hline Nionosers & 3,115 & 3,712 & 3,40 & 4247 & 4,124 & 4,000 \\
\hline 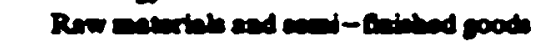 & 1.136 & 1272 & 1.105 & 1,477 & 1,309 & 1,300 \\
\hline Bqpipax & $\infty$ & no & 953 & 1.120 & 1,124 & 1,308 \\
\hline Pood & $\mathbf{4 5 1}$ & 373 & 246 & 300 & 253 & 374 \\
\hline Noneod conomer poods & 876 & 1,157 & 1.113 & 1.345 & 1.393 & 1.578 \\
\hline \multicolumn{7}{|l|}{ 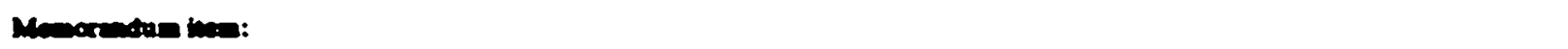 } \\
\hline 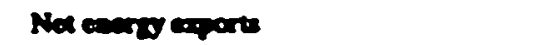 & 100 & $\boldsymbol{\bullet}$ & 3 & 113 & -16 & -25 \\
\hline
\end{tabular}

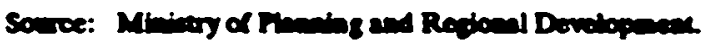

CInternational Monetary Fund. Not for Redistribution 
Table 27. Tuniata: Volume of Forelgn Trade by Commodty Chas, 1990-94 y

(Groweh retes in percent)

\begin{tabular}{|c|c|c|c|c|c|}
\hline & 1990 & 1991 & 1992 & 1993 & $\begin{array}{l}\text { Prel. } \\
\text { Est } \\
1994\end{array}$ \\
\hline Exports, f.o.b. & 4.5 & 4.4 & 3.1 & 0.3 & 11.2 \\
\hline Enersy products & -15.8 & -4.7 & 17.9 & -21.1 & 16.5 \\
\hline Nowenergy & 10.0 & 6.3 & 0.4 & 5.0 & 10.3 \\
\hline Phoephries and derivatives & -13.1 & -3.2 & 9.5 & -9.4 & 9.1 \\
\hline Agriculure (bchuding agro-processing) & 15.8 & 38.7 & -24.4 & 184 & 14.6 \\
\hline Teulles and leather & 26.3 & 4.4 & 84 & 6.8 & 128 \\
\hline Mechanical and electrical industry & 43.2 & 5.9 & -1.6 & 10.2 & 8.5 \\
\hline Otber goods & -320 & -15.2 & -6.4 & -9.6 & -16.1 \\
\hline Imports, c.if. & 9.6 & -7.9 & 15.9 & 26 & 11.4 \\
\hline Energy products & -0.7 & -13.2 & 15.0 & 16.0 & 16.3 \\
\hline Nooenerzy & 10.7 & -7.4 & 16.0 & 1.4 & 10.9 \\
\hline Raw materials and semi-fintahed goods & 4.0 & -8.9 & 24.3 & -7.3 & 3.6 \\
\hline Equipoment & 27.8 & 3.4 & 9.1 & 5.0 & 14.8 \\
\hline Food & -19.1 & -35.5 & 21.9 & 2.3 & 23.4 \\
\hline Nonfood consumer goods & 21.0 & -5.0 & 11.7 & 8.3 & 123 \\
\hline
\end{tabular}

Source: Minimury of Planning and Regional Development.

$y$ Based on customs statistics. 
Table 28. Tunicin: Trede Babance in Hydrocarboos, 1969-93

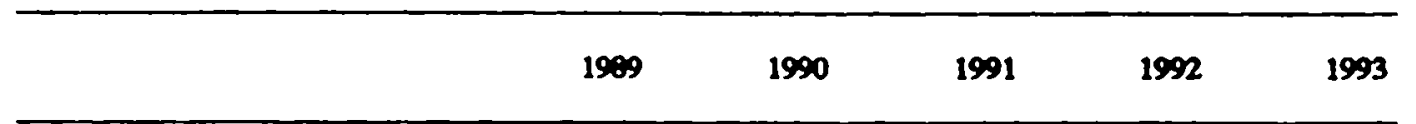

\section{(In minlons of Tuniting diras)}

A. Crude oil

Expors, f.o.b.

Imports, Cab.

Batunce

B. Refined products

Esports, 1.ob.

Imports, f.ab.

Bulance

C. Gas

Epports

Imports

Batance

D. $\operatorname{Total}(A+B+C)$

Exports, 1.0.b.

Imports, f.ob.

Bahoce

543

$-346$

197

$-80$

$$
\begin{array}{r}
506 \\
-67 \\
439
\end{array}
$$

37
-199
-162

53
279

56
-274
-218

50
-258
-208

43
-315

$-272$
$-80$

--
-80
-80

$--\overline{-}$
-65

$-85$

$-85$

$-90$

$-90$

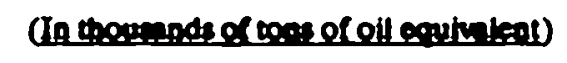

A. Crude oil

Epports

Imports

Balunce

B. Refied products

Exports

Imports

Bapnoce

C. Gas

Expors

Imports

Bubace

D. $\operatorname{Tocal}(A+B+C)$

Exports

Imports

Balance

\section{4,261}

$-553$

3,708
3,257
$-396$
2861

3,304

$-118$

3,186

3,909

$-341$

3,648

3,138

$-295$

2,843

$\begin{array}{rrrrr}100 & 514 & 512 & 160 & 434 \\ -1,554 & -2,008 & -2,186 & -2,254 & -2,521 \\ -1,154 & -1,494 & -1,644 & -1,794 & -2,037\end{array}$

$-1, \overline{337} \quad-1, \overline{019}$

-639
-639

$-1,058$

$-1,126$

$-1,337 \quad-1,019$

$-1,058$

$-1,126$

$\begin{array}{rrrrr}4,661 & 3,771 & 3,846 & 4,449 & 3,572 \\ -3,44 & -3,423 & -2,943 & -3,653 & -3,942 \\ 1,217 & 348 & 903 & 796 & -370\end{array}$

Source: National Instifute of Statiatics and Central Bank of Tuniain. 


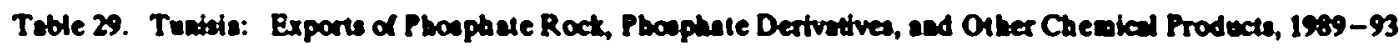

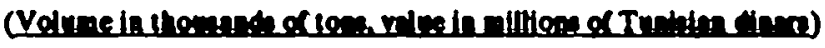

\begin{tabular}{|c|c|c|c|c|c|c|c|c|c|c|}
\hline & \multicolumn{2}{|c|}{$\frac{\text { Volune Value }}{1909}$} & \multicolumn{2}{|c|}{1990} & \multicolumn{2}{|c|}{1991} & \multicolumn{2}{|c|}{1992} & \multicolumn{2}{|c|}{1993} \\
\hline Phraphale rixt & 1,077 & 31 & 593 & 16 & 426 & 11 & 956 & 22 & 1,113 & 27 \\
\hline Superphiephace & 857 & 126 & 751 & 90 & css & o6 & 703 & 95 & 624 & 73 \\
\hline Ammonium phospholes & $\cos$ & 118 & $\operatorname{sos}$ & 81 & s11 & M & 657 & 96 & 718 & 100 \\
\hline Risalcuum phosphate & 47 & 9 & 47 & 8 & 53 & 10 & 34 & 9 & 67 & 12 \\
\hline
\end{tabular}

Souric: Dircetion geacrale des mines 
Table 30. Tunisia: Exports and Imports of Primary Products by Major Categories, 1989-93

(Volume in thousands of tons, value in millions of Tuniaian dinars)

\begin{tabular}{|c|c|c|c|c|c|c|c|c|c|c|}
\hline & Volume & Value & Volume & Value & Volume & Value & Volume & Value & Volume & Value \\
\hline & \multicolumn{2}{|c|}{1989} & \multicolumn{2}{|c|}{1990} & \multicolumn{2}{|c|}{1991} & \multicolumn{2}{|c|}{1992} & \multicolumn{2}{|c|}{1993} \\
\hline \multicolumn{11}{|l|}{ Exports } \\
\hline Olive oil & 47 & 82 & 50 & 107 & 158 & 267 & 97 & 139 & 123 & 177 \\
\hline Dates & 17 & 38 & 18 & 45 & 20 & 49 & 17 & 44 & 19 & 48 \\
\hline Almonds & -- & - & -- & 1 & 1 & 2 & 1 & 2 & 1 & 4 \\
\hline Citrue & 41 & 13 & 29 & 10 & 26 & 11 & 20 & 8 & 24 & 10 \\
\hline Wine $y$ & 8 & 4 & 9 & 4 & 11 & 17 & 9 & 11 & 9 & 13 \\
\hline Fish & 14 & 84 & 17 & 101 & 14 & 80 & 12 & 69 & 16 & 90 \\
\hline \multicolumn{11}{|l|}{ Imports } \\
\hline Soft wheat & 611 & 105 & 714 & 94 & 559 & 48 & 596 & 72 & 686 & 86 \\
\hline Hard wheat & 545 & 100 & 184 & 30 & 118 & 16 & 57 & 8 & 29 & 5 \\
\hline Barley & 177 & 24 & 128 & 17 & 4 & 1 & 5 & 1 & 30 & 3 \\
\hline Maize & 251 & 35 & 293 & 36 & 238 & 30 & 343 & 38 & 290 & 37 \\
\hline Sugar & 176 & 47 & 208 & 71 & 165 & 43 & 235 & 59 & 213 & 62 \\
\hline Tea & 9 & 12 & 11 & 16 & 13 & 18 & 14 & 19 & 11 & 17 \\
\hline Milk & 29 & 46 & 30 & 43 & 17 & 21 & 28 & 41 & 38 & 42 \\
\hline Soybean oil & 83 & 34 & 84 & 35 & 56 & 24 & 106 & 40 & 117 & 54 \\
\hline \multicolumn{11}{|l|}{ Meat (including } \\
\hline live animals for slaughter) & 16 & 21 & 14 & 24 & 13 & 18 & 14 & 21 & 15 & 29 \\
\hline
\end{tabular}

Sources: Central Bank of Tunisia; and National Statistice Institute.

1/ Includes other alcoholic beverages. 


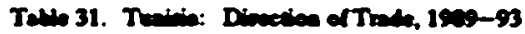

(1....:日)

\begin{tabular}{|c|c|c|c|c|c|}
\hline & 190 & 1990 & 1991 & 192 & $19 \sin$ \\
\hline \multicolumn{6}{|l|}{ Biper, lal } \\
\hline 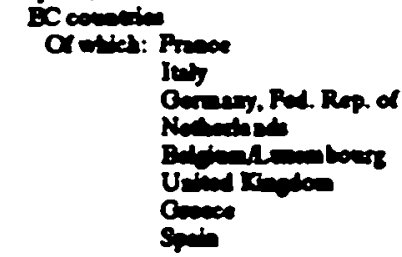 & $\begin{array}{l}73.7 \\
246 \\
129 \\
126 \\
25 \\
44 \\
14 \\
3.7 \\
32\end{array}$ & $\begin{array}{l}718 \\
256 \\
212 \\
15.1 \\
25 \\
7.0 \\
1.6 \\
0.4 \\
2.6\end{array}$ & $\begin{array}{r}76.6 \\
2 \$ 2 \\
19.7 \\
16.4 \\
27 \\
6.3 \\
13 \\
0.6 \\
3.7\end{array}$ & $\begin{array}{r}7.4 \\
272 \\
172 \\
17.1 \\
26 \\
69 \\
18 \\
21 \\
28\end{array}$ & $\begin{array}{l}79.1 \\
30.0 \\
16.3 \\
17.4 \\
3.1 \\
7.3 \\
13 \\
0.1 \\
2.5\end{array}$ \\
\hline Am Manter & $\begin{array}{l}7.1 \\
42 \\
2.2 \\
0.6 \\
0.1\end{array}$ & $\begin{array}{l}7.7 \\
4.6 \\
2.3 \\
0.7 \\
0.1\end{array}$ & $\begin{array}{l}2.4 \\
5.5 \\
2.1 \\
0.7 \\
0.1\end{array}$ & $\begin{array}{l}7.9 \\
4.4 \\
24 \\
1.1 \\
--\end{array}$ & $\begin{array}{l}76 \\
50 \\
18 \\
29 \\
--\end{array}$ \\
\hline Orwel: Uh & $\begin{array}{l}192 \\
23 \\
\text { as } \\
\text { of } \\
02 \\
03\end{array}$ & $\begin{array}{l}145 \\
0.9 \\
0.5 \\
13 \\
03 \\
0.4\end{array}$ & $\begin{array}{l}150 \\
0.7 \\
0.7 \\
1.1 \\
0.3 \\
0.5\end{array}$ & $\begin{array}{l}13.7 \\
0.8 \\
0.9 \\
0.3 \\
0.3 \\
0.3\end{array}$ & $\begin{array}{l}13.3 \\
0.7 \\
1.3 \\
02 \\
02 \\
0.5\end{array}$ \\
\hline Toed & 1000 & 100.0 & 1000 & 1000 & 1000 \\
\hline \multicolumn{6}{|l|}{ inpros cil } \\
\hline Shes of & $\begin{array}{l}60 \\
214 \\
137 \\
11.7 \\
24 \\
4.7 \\
1.5 \\
1.9 \\
3.3\end{array}$ & $\begin{array}{l}202 \\
27.9 \\
159 \\
125 \\
26 \\
4.8 \\
1.7 \\
0.1 \\
3.1\end{array}$ & $\begin{array}{l}\pi .7 \\
260 \\
174 . \\
143 \\
22 \\
5.4 \\
1.8 \\
0.8 \\
3.1\end{array}$ & $\begin{array}{r}71.1 \\
285 \\
182 \\
140 \\
22 \\
4.7 \\
18 \\
06 \\
33\end{array}$ & $\begin{array}{r}76 \\
268 \\
112 \\
130 \\
22 \\
43 \\
23 \\
08 \\
32\end{array}$ \\
\hline 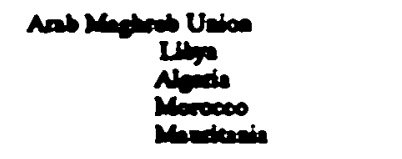 & $\begin{array}{l}4.4 \\
0.4 \\
2.4 \\
10 \\
0.6\end{array}$ & $\begin{array}{l}34 \\
0.5 \\
20 \\
0.9 \\
--\end{array}$ & $\begin{array}{l}3.7 \\
0.6 \\
1.9 \\
1.2 \\
--\end{array}$ & $\begin{array}{l}41 \\
08 \\
24 \\
12 \\
--\end{array}$ & $\begin{array}{l}32 \\
08 \\
16 \\
\text { as } \\
--\end{array}$ \\
\hline 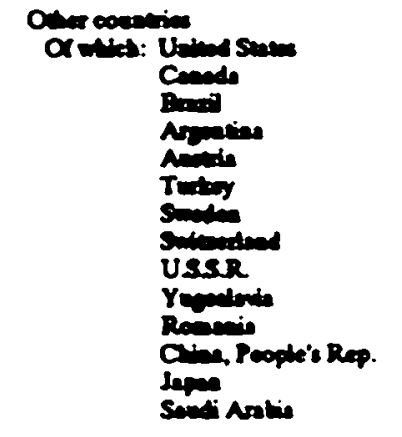 & $\begin{array}{l}2.7 \\
58 \\
1.1 \\
0.3 \\
0.5 \\
0.7 \\
1.5 \\
1.0 \\
1.7 \\
0.8 \\
0.4 \\
0.7 \\
0.4 \\
1.4 \\
0.3\end{array}$ & $\begin{array}{l}264 \\
5.2 \\
0.9 \\
0.5 \\
0.6 \\
0.6 \\
0.9 \\
1.0 \\
12 \\
1.7 \\
0.7 \\
0.1 \\
0.6 \\
1.8 \\
0.8\end{array}$ & $\begin{array}{l}24.6 \\
1.8 \\
1.6 \\
0.4 \\
0.7 \\
0.6 \\
1.1 \\
1.3 \\
1.4 \\
1.4 \\
0.4 \\
0.2 \\
0.7 \\
2.3 \\
0.5\end{array}$ & $\begin{array}{l}24.5 \\
50 \\
10 \\
0.6 \\
\text { as } \\
0.5 \\
0.9 \\
1.1 \\
12 \\
1.3 \\
0.5 \\
0.1 \\
0.8 \\
23 \\
0.3\end{array}$ & $\begin{array}{l}282 \\
58 \\
0.5 \\
0.7 \\
0.6 \\
0.4 \\
0.5 \\
1.3 \\
13 \\
16 \\
0.1 \\
0.0 \\
0.5 \\
2.3 \\
0.3\end{array}$ \\
\hline Totel & 1000 & 1000 & 1000 & 1000 & 1000 \\
\hline
\end{tabular}

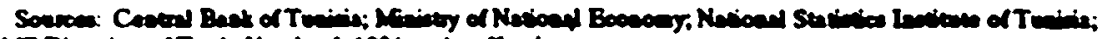

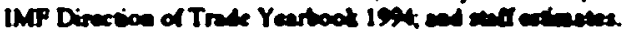


Table 32. Tunisia: External Debt and Debt Service Payments, 1989-93

\begin{tabular}{|c|c|c|c|c|c|}
\hline & 1989 & 1990 & 1991 & $\frac{\text { Prel. }}{1992}$ & $\frac{\text { Prel. }}{1993}$ \\
\hline & \multicolumn{5}{|c|}{ (In millions of SDRs) } \\
\hline $\begin{array}{l}\text { Disbursements } \\
\text { Long-term capital } \\
\text { Medium-term capital }\end{array}$ & $\begin{array}{l}700 \\
485 \\
215\end{array}$ & $\begin{array}{l}700 \\
478 \\
222\end{array}$ & $\begin{array}{l}805 \\
542 \\
263\end{array}$ & $\begin{array}{l}816 \\
482 \\
334\end{array}$ & $\begin{array}{l}881 \\
558 \\
323\end{array}$ \\
\hline $\begin{array}{l}\text { Debt service payments } \\
\text { Medium- and long-term } \\
\text { Interest } \\
\text { Principal }\end{array}$ & $\begin{array}{l}900 \\
302 \\
598\end{array}$ & $\begin{array}{l}909 \\
289 \\
620\end{array}$ & $\begin{array}{l}895 \\
280 \\
615\end{array}$ & $\begin{array}{l}927 \\
298 \\
629\end{array}$ & $\begin{array}{l}948 \\
338 \\
610\end{array}$ \\
\hline $\begin{array}{l}\text { IMF } \\
\text { Charges } \\
\text { Repurchases }\end{array}$ & $\begin{array}{r}20 \\
20 \\
--\end{array}$ & $\begin{array}{r}104 \\
22 \\
82\end{array}$ & $\begin{array}{r}113 \\
14 \\
99\end{array}$ & $\begin{array}{l}36 \\
15 \\
21\end{array}$ & $\begin{array}{r}17 \\
13 \\
4\end{array}$ \\
\hline $\begin{array}{l}\text { Total debt service } \\
\text { Interest } \\
\text { Principal }\end{array}$ & $\begin{array}{l}921 \\
322 \\
598\end{array}$ & $\begin{array}{r}1013 \\
311 \\
702\end{array}$ & $\begin{array}{r}1008 \\
294 \\
715\end{array}$ & $\begin{array}{l}963 \\
313 \\
650\end{array}$ & $\begin{array}{l}965 \\
351 \\
614\end{array}$ \\
\hline $\begin{array}{l}\text { Debt outstanding } \\
\text { Medium- and long-term } \\
\text { Arab Monetary Fund } \\
\text { Fund credit }\end{array}$ & $\begin{array}{r}4,713 \\
4,500 \\
7 \\
206\end{array}$ & $\begin{array}{r}5,007 \\
4,881 \\
3 \\
124 \\
\\
\text { (In }\end{array}$ & $\begin{array}{r}5,355 \\
5,175 \\
-- \\
180 \\
\\
\text { of cur }\end{array}$ & $\begin{array}{r}5,424 \\
5,202 \\
11 \\
211 \\
\\
\text { ceipts) }\end{array}$ & $\begin{array}{r}5,637 \\
5,422 \\
8 \\
207\end{array}$ \\
\hline $\begin{array}{l}\text { Total debt service } \mathbf{y} \\
\text { Interest } \\
\text { Principal }\end{array}$ & $\begin{array}{r}23.2 \\
8.1 \\
15.1\end{array}$ & $\begin{array}{r}23.2 \\
7.1 \\
16.1\end{array}$ & $\begin{array}{r}23.8 \\
6.9 \\
16.9\end{array}$ & $\begin{array}{r}20.2 \\
6.6 \\
13.7\end{array}$ & $\begin{array}{r}20.5 \\
7.5 \\
13.1\end{array}$ \\
\hline $\begin{array}{l}\text { Excluding IMF } \\
\text { Interest } \\
\text { Principal }\end{array}$ & $\begin{array}{r}22.7 \\
7.6 \\
15.1\end{array}$ & $\begin{array}{r}20.8 \\
6.6 \\
14.2 \\
\text { (In }\end{array}$ & $\begin{array}{r}21.2 \\
6.6 \\
14.6 \\
\text { tof GD } \\
\end{array}$ & $\begin{array}{r}19.5 \\
6.3 \\
13.2\end{array}$ & $\begin{array}{r}20.1 \\
7.2 \\
13.0\end{array}$ \\
\hline Debt outstanding & 58.8 & 55.2 & 55.2 & 51.5 & 55.2 \\
\hline Total debt service & 11.8 & 11.2 & 10.6 & 8.7 & 9.2 \\
\hline
\end{tabular}

Sources: Ministry of Planning and Regional Development; Central Bank of Tunisia; and IMF, Treasurer's Department.

1/ Includes IMI: charges and repurchases. 
Table 33. Tunitia: Compocilioe of External Debl by Credilor, 1989-93

(In millions of Tuniaine dinars end of period)

\begin{tabular}{|c|c|c|c|c|c|}
\hline & 1939 & 1990 & 1991 & $\frac{\text { Pret }}{1992}$ & $\frac{\text { Pret. }}{1993}$ \\
\hline $\begin{array}{l}\text { Muttiateral organimtions } \\
\text { Of whict: Worid Bant } \\
\text { Africas Development Bank }\end{array}$ & $\begin{array}{r}1,370 \\
987 \\
235\end{array}$ & $\begin{array}{r}1,610 \\
1,120 \\
395\end{array}$ & $\begin{array}{r}1,944 \\
1,230 \\
587\end{array}$ & $\begin{array}{r}2,015 \\
1,206 \\
668\end{array}$ & $\begin{array}{l}2,936 \\
1,571 \\
1,056\end{array}$ \\
\hline $\begin{array}{l}\text { OECD courntries } \\
\text { Of which: France } \\
\cdot \text { Gerwany } \\
\text { Jepan } \\
\text { United Sutes } \\
\text { Italy }\end{array}$ & $\begin{array}{r}3,064 \\
762 \\
611 \\
548 \\
302 \\
202\end{array}$ & $\begin{array}{r}3,363 \\
841 \\
639 \\
531 \\
426 \\
268\end{array}$ & $\begin{array}{l}3,662 \\
963 \\
667 \\
534 \\
505 \\
318\end{array}$ & $\begin{array}{r}3,855 \\
970 \\
683 \\
551 \\
512 \\
404\end{array}$ & $\begin{array}{r}3,872 \\
1,109 \\
681 \\
638 \\
628 \\
464\end{array}$ \\
\hline $\begin{array}{l}\text { Arab countries and organteations } \\
\text { Of whict: Saudi Fund } \\
\text { Tunill Fund } \\
\text { Arab Fuod for Boonomic } \\
\text { and Soctal Developonent }\end{array}$ & $\begin{array}{l}432 \\
121 \\
134 \\
93\end{array}$ & $\begin{array}{l}467 \\
133 \\
145 \\
100\end{array}$ & $\begin{array}{l}567 \\
134 \\
144 \\
143\end{array}$ & $\begin{array}{l}64 \\
147 \\
130 \\
219\end{array}$ & $\begin{array}{l}825 \\
125 \\
127 \\
233\end{array}$ \\
\hline Otber countries & 87 & 100 & 88 & 98 & 40 \\
\hline Finnectal martets & 39 & 270 & 140 & 188 & 122 \\
\hline Total medium - and bong-term debt & 5,350 & 5,810 & 6,400 & 6,800 & 7,734 \\
\hline $\begin{array}{l}\text { Arab Mosetary Fund } \\
\text { Internatboal Mosetary Fund }\end{array}$ & $\begin{array}{r}8 \\
245\end{array}$ & $\begin{array}{r}3 \\
147\end{array}$ & $-\overline{203}$ & $\begin{array}{r}14 \\
276\end{array}$ & $\begin{array}{r}12 \\
298\end{array}$ \\
\hline Total external debe & 5,603 & 5,960 & 6,622 & 7,090 & 8,104 \\
\hline (as percent of GDP) & 58.8 & 55.2 & $\mathbf{5 5 . 2}$ & 51.5 & 55.2 \\
\hline
\end{tabular}

Sources: Miniatry of Planating and Regional Development; Central Bank of Tunitia; and staff eatimates. 
Table 34. Tunisia: Selected Exchange Rate Indices, 1989-94 1/

(Period averages; $1980=100)$

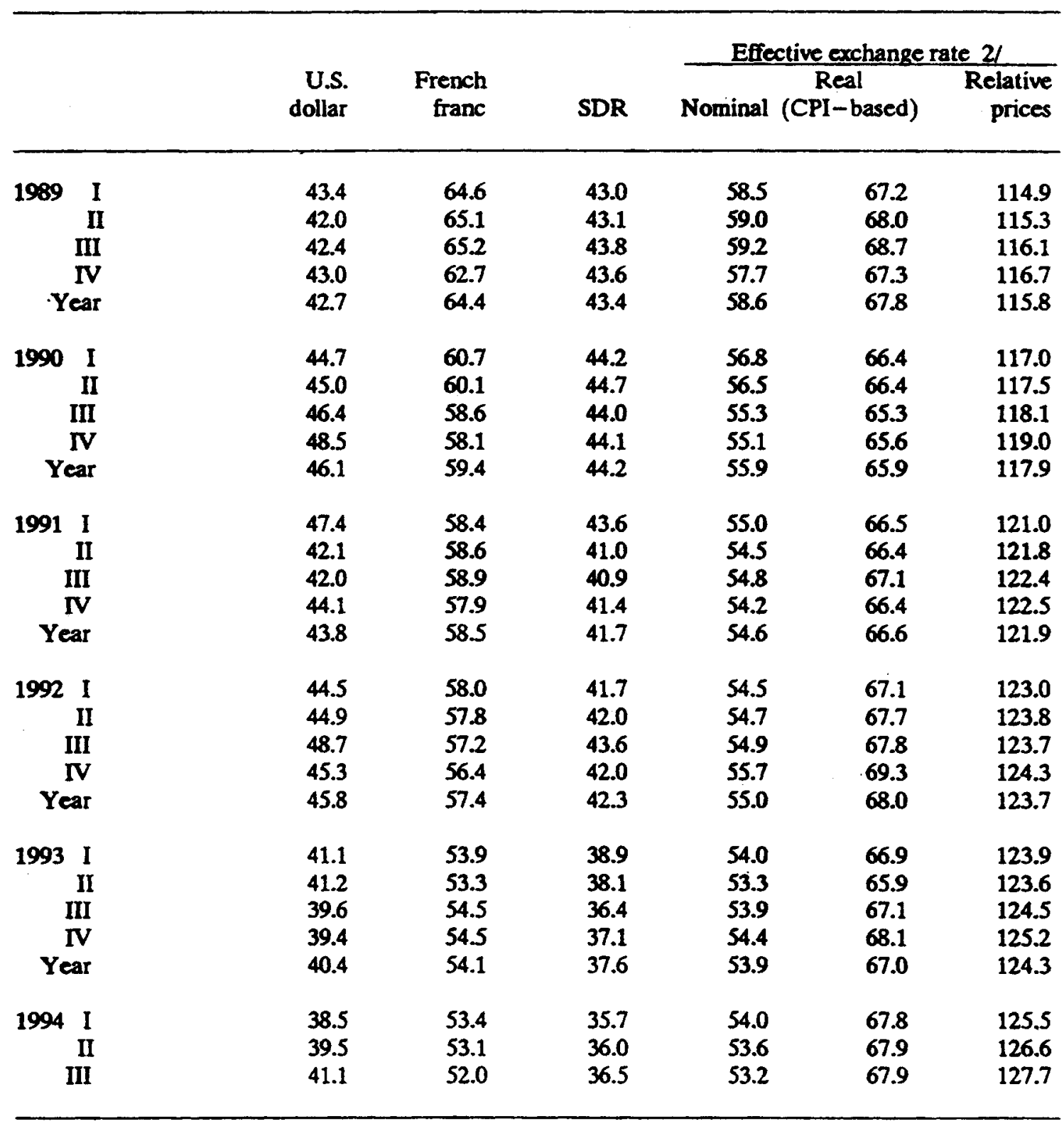

Sources: IMF, Data Fund, and Information Notice System.

1/ Foreign currency units per Tunisian dinar.

2/ Weighted by non-oil trade and tourism flows of 16 partner and competitor countrics; (-) denotes depreciation. 
Table 35. Tunisia: Structure of Imports by Regime, 1989-92 1/

(In millions of Tunisign dinars)

\begin{tabular}{lrrrr}
\hline & 1989 & 1990 & 1991 & $\frac{\text { PreL }}{1992}$ \\
\hline Regime with restrictions 2/ & 1,152 & 1,133 & 1,074 & 534 \\
Import licemse 3/ & 878 & 951 & 954 & 446 \\
Annual import authorization 4 y & 274 & 182 & 120 & 88 \\
Regime without restrictions & 1,974 & 2,479 & 2,484 & 3,024 \\
$\begin{array}{l}\text { Import certificate 5/ } \\
\text { Of which: equipment goods }\end{array}$ & 1,654 & 2,264 & 2,311 & 2,851 \\
Temporary admission & 150 & 146 & 166 & 166 \\
Total $1 /$ & 320 & 215 & 173 & 173 \\
\hline
\end{tabular}

Source: Central Bank of Tunizia.

1) Total imports are less than those recorded in the balance of payments, primarily because imports without payments are not covered by the above regimes.

2f Quantitative restrictions.

3/ Import licenses, which are issued for six months, authorize the importation of certain consumer goods, including durable goods.

4) Under an annual import authorization, certain staples or industrial products can be imported by specific industrial enterprises, agencies, or merchants up to a giobal annual limit, expreased in dinars.

5/ Document required for the import of liberalized goods can be obtained upon presentation of a commercial contract, domiciled with an authorized intermediary. 
Tunisia: Summary of the Tax System as of September 30, 1994

(All amounts in Tunisien diners)

\section{Tax}

$\operatorname{Tax}$

Nature of Iax

Exemptions and Deductions

Rates

1. Taxes on net income and profits

1.1 Corporations

Code de L'impot sur le revenu des personnes physiques et de 1 'impot sur les sociétés (CIRPPIS), January 5, 1990, 1/

Levied on all resident corporations, cooperatives, and autonomous public entities engaged in industrial or conmerciel activity, as well on Tunisiansource profits of nonresident companies.

Losses can be carried forward as a deduction against profits of the following three years.

Provisional advance peyments are required on current year's profits, in three installments each being equel to 30 percent of the previous year's income tax in the $6 \mathrm{th}$, $9 \mathrm{th}$, and $12 \mathrm{th}$ months of their taxable year. Adjustment is made upon submission of the tax return for the taxable year.
Exemptions: nonprofit professional associations with revenue from tax or quasi-tax sources; mutual funds; savings and assistance funds managed by nonpaid officials; nonproftt. public entities; service cooperatives in agricultural and fishing activities; employee-owned production cooperatives.

Deductions: linear or degressive depreciations are allowed for business fixed assets; provisions for bad debts up to 10 percent of taxable profits; deduction of up to 35 percent of net profits for purchase of newly lesued equity instruments in enterprises

benefiting from the incentives under the Investment Incentive Law (Lan No. 93-120 of Decenber 1993); deductions in various degrees of income from activities encouraged by the Investment Incentive Law; deduction allowed for charitable donations up to 2 percent of the turnover and for representation expenses up to 1 percent of the turnover with a maximum of $D$ 20,$000 ; 50$ percent of the construction cost of housing built for employees is deductible over a period of ten years.
Normal rate: 35 percent.

Reduced rate: 10 percent. The latter is applicable to profits of the following companies: onterprises in handicraft, agricultural, and fishing ectivities; service and

consumption cooperatives; profits from youth employment programs or promotion funds for handicrafts and small traders. Exemptions cannot reduce tax liability below 10 percent.

Minimum tax: 0.5 percent of the turnover of the taxable year, with a celling of $D 500$ for companies subject to 10 percent rate and $D$ 1,000 for companies subject to 35 percent rate. Tax withholding at source is applied to wages,

salaries, interest, comissions,

brokerage fees, royalties, and rents. 


\section{(All mounts in Tunisien diners)}

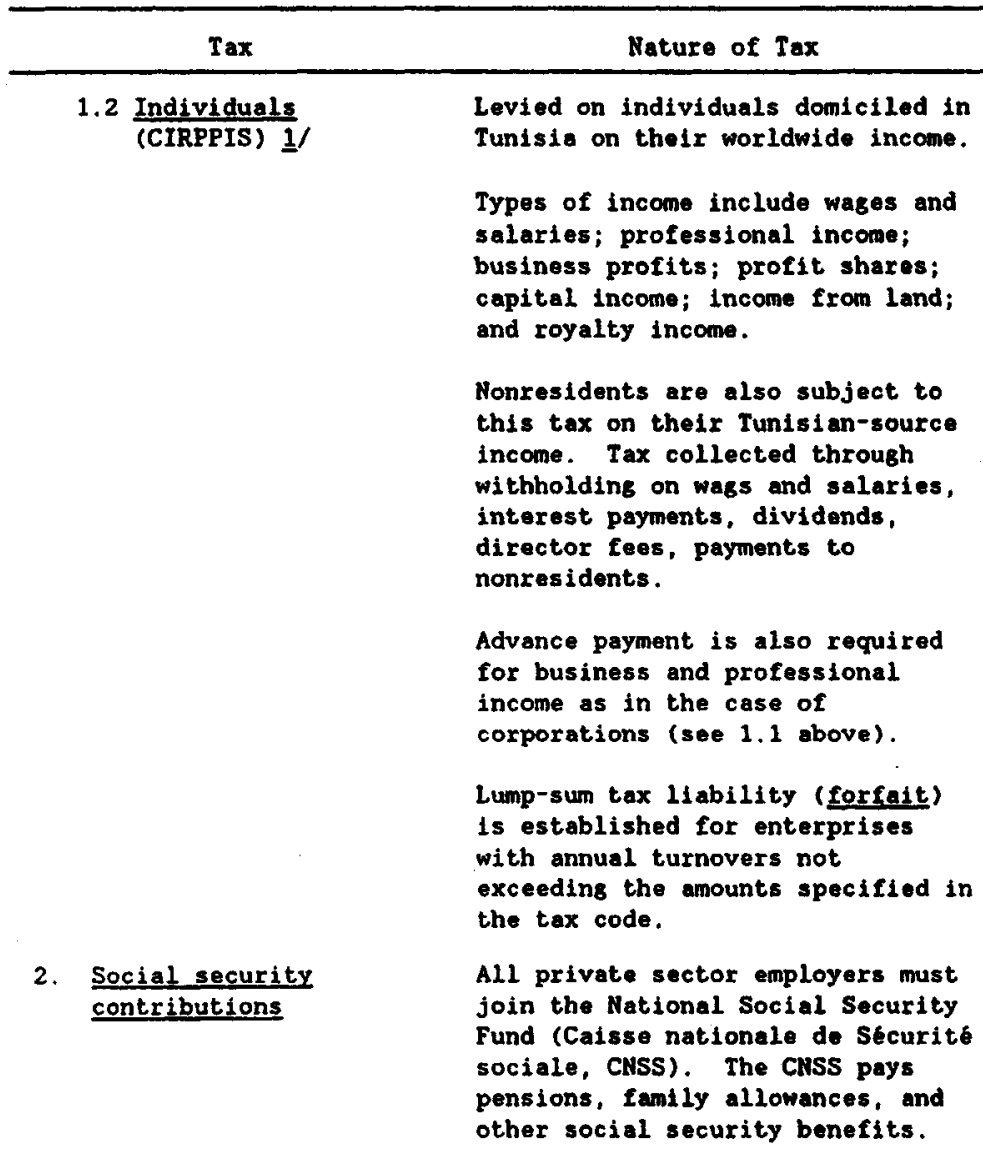

3. Taxes on payroll and work force

\section{Exemptions and Deductions}

Exemptions: remunerations of

foreign diplomats (exempted on a reciprocal basis); life annulties pald to work-related accident victims; indonnities paid to victims of physical attecks by virtue of a court verdict; social security and welfare payments; special allowances to cover workrelated expenses; dividends pald by entities subject to the corporate tax; interest income from savings deposits linked to financing for bousing; interest from bank deposits in foreign currency or convertible dinars; interest income from special savings accounts with banks and the National Savings Fund of Tunisia, and bonds up to D 1,500, with limit of D 1,000 for interest from special savings accounts.

Deductions: same as in the case of corporate profits (see 1.1 above).

Exemptions: none.
Rates

Brackets

First 1,500

Next 3,500

- 5,000

-10,000

- 30,000

Over 50,000

Minimum tax: 0.5 percent of the turnover of the taxable yeax with a colling of D 500. Deductions and exemptions cannot lower tax liability below 30 percent of tax due in the absence of deductions and exemptions.

(In percent)

$2 e r o$
15
20
25
30
35

cent of the
Deductions
lower tax
dent of tax
deductions

Social security

Employers: 15 percent.

Employees: 6 percent.

Retirement

Enployers: 2.5 percent.

Employees: 1.25 percent.

Normal rate: 2 percent

Reduced rate: 1 percent

(for industrial manufacturing

jobs).
Deductions: none. 
(Alt nopere in Tralelen diners)

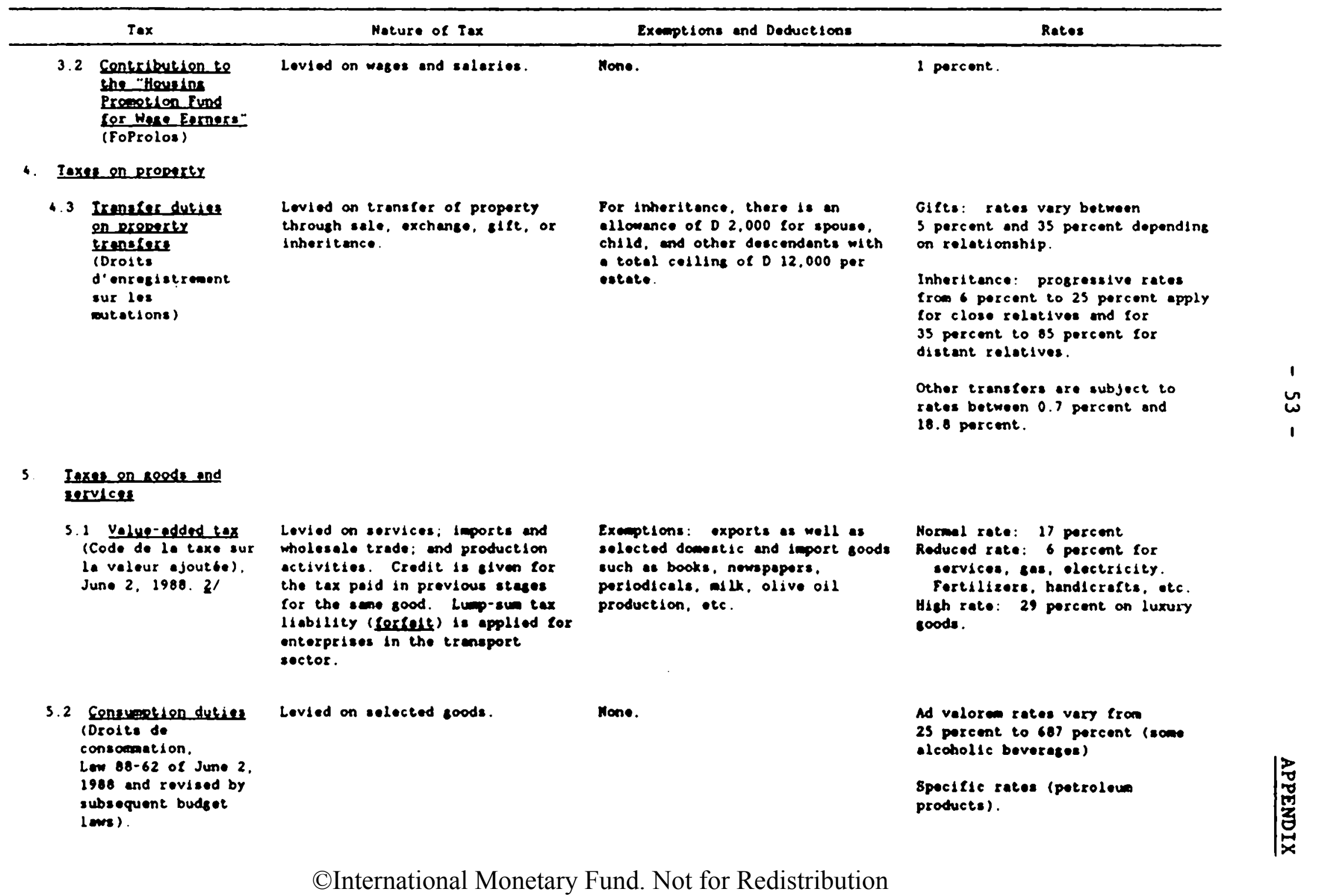


Tunisle: Swaery of the Iax Systen es of 8opteber 30, 1994

(All moonts in Tuntein diners)

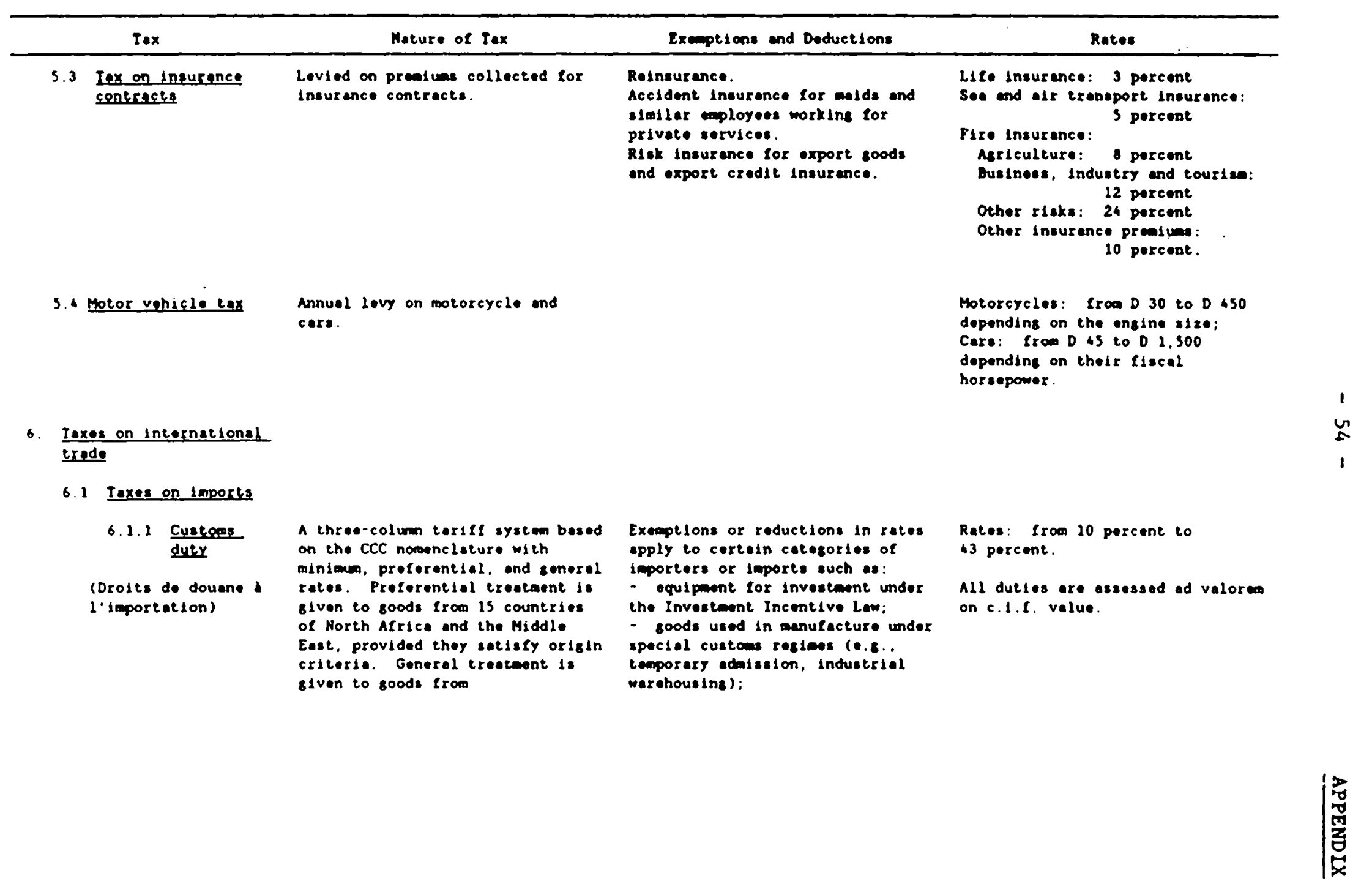


(Al1 amounts in Tunisten diners)

Tax Nacure of $\operatorname{Tax}$

Excaptions and Deductions

Rates

countries that do not have special commercial relationships with Tunisie, or do not apply the mostfavored nation clause.

6.1 .2 Complementary duty on imports (Droit

complémentaire à 1 'importation)

6.1 .3 Service fee on imports (Redevance sur prestations douanières à

1'importation).
Additional temporary import duty

Levied on limited range of

finished and semifinished goods

produced locally for which imports are liberalized.

Flat fiscal duty on imports, regardless of origin.
- coods used by particular institutions and bodies; military corces, diplomatic persomel, and International oreanizations.

eirlines, etc.:

- goods used for educational. cultural, social and health purposes;

- housohold effects and travel goods within specified limits.

\section{Exemptions or deductions may also \\ be applied to certain categories \\ of foodstuffs imported under \\ special temporary tax relief \\ (résimes fiscaux_privilegiés \\ conjoncture1s).}

Rates: from 10 percent to 30 percent. To be eliminated gradually over three years from the date of introduction.

Exemptions: numerous.

2 percent of the total of import duties, including VAT, excises, and compensatory duty, with a minimum of D 5 per declared item.
1 
Tunisia: Sumary of the Tax System as of September 30, 1994

(All amounts in Iunision dinars)

\begin{tabular}{|c|c|c|c|c|}
\hline & $\operatorname{Tax}$ & Nature of Tax & Exemptions and Deductions & Rates \\
\hline \multicolumn{5}{|c|}{6.2 Export duties } \\
\hline 6.2 .1 & $\begin{array}{l}\text { Service fee on } \\
\text { exports } \\
\text { (Redevances } \\
\text { sur prestations } \\
\text { doumières à } \\
\text { l'exportation). }\end{array}$ & $\begin{array}{l}\text { Flat fiscal duty on selected } \\
\text { exports. }\end{array}$ & Exemptions: numerous. & $\begin{array}{l}1.5 \text { percent of the export value } \\
\text { (f.o.b.). Budget Law of } 1988 \\
\text { provides a list of taxable } \\
\text { exports. }\end{array}$ \\
\hline \multicolumn{5}{|c|}{ Other taxes } \\
\hline 7.3 .2 & $\begin{array}{l}\text { Stamp duties } \\
\text { (Droits de } \\
\text { timbre). }\end{array}$ & $\begin{array}{l}\text { Specific duties on a wide range of } \\
\text { civil, administrative, and } \\
\text { judicial procedures. }\end{array}$ & Exemptions: numerous. & $\begin{array}{l}\text { Various rates between } D 0.2 \text { and } \\
\text { D } 100 \text {. }\end{array}$ \\
\hline
\end{tabular}

Sources: Code de 1 impôt sur le revenu des personnes physiques et de l.jmpót sur les societes; Code de la taxe sur la valeur ajoutée; and Code de la douane.

1/ Applicable to 1990 income and profits.

2/ Effective July 1, 1988. 